\title{
DELINEAMENTO GUADALUPE PARA TRÊS FATORES, ANALISADO ATRAVES DE MODELO DE REGRESSĀO POLINOMIAL QUADRÁTICA
}

JOASSY DE PAULA NEVES JORGE

Orientador: PROF. FREDERICO PIMENTEL GOMES

Dissertação apresentada à Escola Superior de Agricultura "Luiz de Queiroz", da Universidade de São Paulo, para obtenção do título de Mestre em Estatística e Experimentação Agronômica.

PIRACICABA

Estado de São Paulo - Brasil

Junho, 1980 
ii.

A meus PAIS, por nos terem criado num lar feliz 


\section{AGRADECIMENTOS}

Ao Dr. Frederico Pimentel Gomes, Professor Catedrático do Departomento de Matemática e Estatistica da ESALQ, pela solicitude e minucioso cuidado com que foi orientador deste trabalho.

Ao Corpo Docente do Departomento de Matemätica e Estatistica da ESALQ, pelos ensinomentos ministrados durante o curso de pós-graduação.

Ao Dr. Armando Conagin, incentivador e mestre de nosso trabalho realizado no Instituto Agronômico de Compinas.

Ao Conselho Nacional de Desenvolvimento Cientifico $e$ Tecnológico, por colaborar em nosso desempenho profissional durante oito anos.

Aos companheiros no curso de pós-graduação, que, de colegas, se transformarom em amigos.

Aos funcionários do Departomento de Matemática e Estatistica da ESALQ, pela colaborasão prestada sempre.

As Srtas. Maria Inês Corat e Liliana Solha, do Instituto Agronômico de Campinas, pelo esmero com que realizaram o trabalho de datilografia e desenho.

A todos que, mesmo sem o saberem, auxiliaram a levar a termo o projeto de pós-graduasão. 


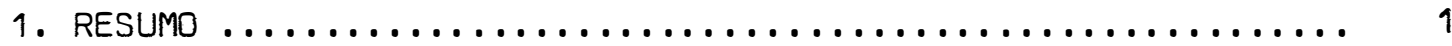

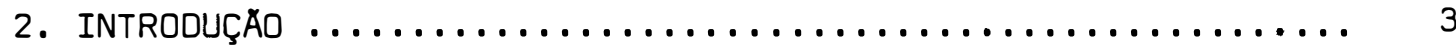

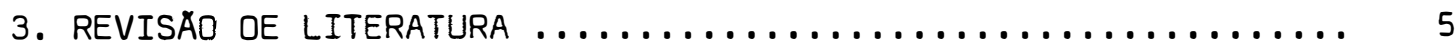

3.1. Generalidades $\ldots \ldots \ldots \ldots \ldots \ldots \ldots \ldots \ldots \ldots \ldots \ldots \ldots \ldots, 5$

3.2. O delineamento Guadalupe $\ldots \ldots \ldots \ldots \ldots \ldots \ldots \ldots \ldots \ldots, 12$

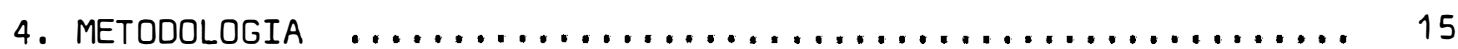

4.1. Características do delineamento Guadalupe para três fa-

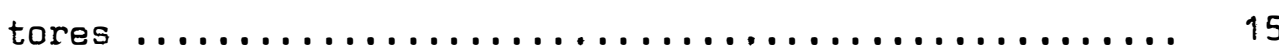

4.2. Metodologia de superfície de resposta $\ldots \ldots \ldots \ldots \ldots \ldots, 17$

4.3. Modelo de regressão linear: cálculo das estimativas dos parâmetros e de suas variâncias e covariâncias; anälise da variància e testes de hipótese; intervalos de confian

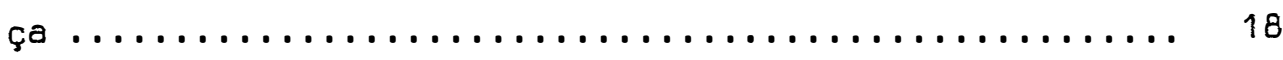

4.4. Aplicação do modelo de regressão linear polinomial quadrätica ao delineamento Guadalupe com três fatores ..... 22

5. ORTOGONALIZAÇÃO DO DELINEAMENTO GUADALUPE COM TRES FATORES .. 28

5.1. Generalidades $\ldots \ldots \ldots \ldots \ldots \ldots \ldots \ldots \ldots \ldots \ldots \ldots \ldots, 28$

5.2. Aplicação de modelo de regressão polinomial quadrática, com variāvel reduzida ....................... 29

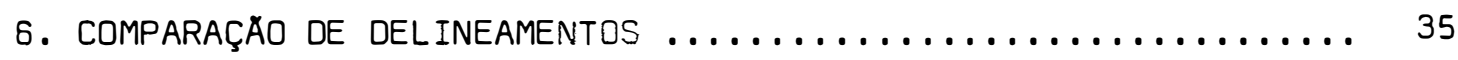

6.1. Eficiência do delineamento Guadalupe em relação aos delineamentos fatorial $3 \times 3 \times 3$ e ao composto central origi -

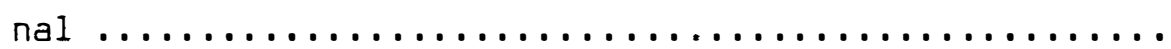


Păg.

6.2. Eficiéncia do delineamento Guadalupe ortogonal em relação ao Guadalupe original, ao fatorial $3 \times 3 \times 3$ e ao composto central original ..................... 38

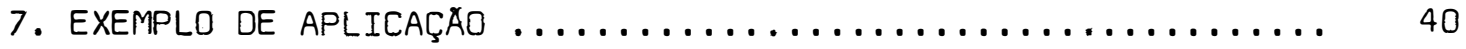

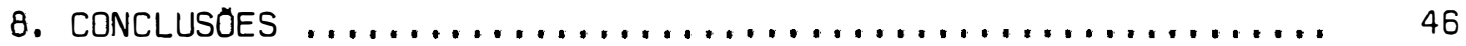

8.1. Delineamento Guadalupe original para trēs fatores ..... 46

8.2. Delineamento Guadalupe ortogonal para trés fatores .... 47

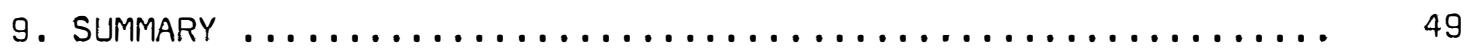

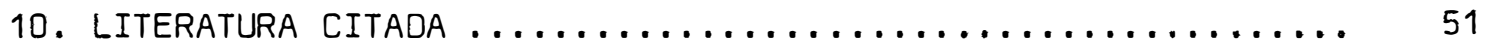




\section{RESUMO}

Com R.A. Fisher iniciou-se a procura de delineamentos, tanto experimentais como de tratamentos, que possibilitassem estudo mais eficiente de respostas à variação de fatores aplicados a determinado fenômeno. Introduzidos inicialmente em ensaios agronōmicos, os delineamentos adaptam-se a vários ramos de pesquisa.

Além de utilizar os ensaios fatoriais e os fatoriais fracionados, no campo da experimentação industrial estão-se desenvolvendo, desde os anos 50, os delineamentos compostos centrais e delineamentos rotacionais; eles também vèm sendo estendidos a outros campos de pesquisa. Na presente dissertação aplicou-se a metodologia de superfície de resposta a um delineamento do tipo composto central, denominado Guadalupe, para trés fatores em sete níveis equidistantes, que vão de zero a seis, num total de vinte e sete pontos experimentais. Consta de um ponto central, dezoito axiais e oito fatoriais, cujas projeções sobre os eixos estão nos extremos do intervalo. A análise utilizou um modelo de regressão polinomial quadrática, com componentes ortogonais de $1^{8}$ e de $2^{\circ}$ grau. 
São dados: o cảlculo das estimativas dos parämetros do modelo, de suas variàncias e covariànciası a análise da vartâncta 8 os testes de hipótese; os intervalos de confiança para os parämetrós e as respostas esperadas. A seguir vem sua aplicação ao delineamento Guadalupe.

Em comparação com os delineamentos fatorial $3 \times 3 \times 3$ e composto central original, tradicionais em pesquisa agronômica e industrial, ele se revelou menos eficiente que o fatorial $3 \times 3 \times 3$ e mais que o composto central, para ensaios agrícolas, e mais eficiente que os dols no 80 . gundo caso, de acordo com critérios preconizados por PIMENTEL GOMES CAMPOS (1972) e por BOX e WILSON (1951), respectivamente.

0 fato de os componentes quadräticos puros estarem correlacionados levou a autora à procura de novos níveis, para cada fator, que ortogonalizassem o delineamento Guadalupe, utilizando um modelo de regressão polinomial quadrática. Os seis níveis dos pontos axiais -continuaram equidistantes entre si e ao ponto central; mas surgiram dois outros niveis por fator, correspondentes à projeção, sobre os eixos, dos nÍveis dos pontos fatoriais, internos ao intervalo total.

Pelos mesmos critérios de comparação, o Guadalupe ortogonal é menos eficiente que o Guadalupe original e o fatorial $3 \times 3 \times 3$, e mais que o composto central original, em ensaios de adubação; mas é mais eficiente que os mesmos, na estimação dos componentes quadräticos puros, em pesquisa industrial ou afins.

Foi simulado um experimento de adubação $\operatorname{com} N, P$ e , em milho, como exemplo da utilização do delineamento Guadalupe no campo agronômico, com anālise que aplica a metodologia usada no presente trabalho. 


\section{INTRODUÇÃO}

Os avanços da ciência e da tecnologia se fazem utilizando experimentos sempre mais detalhados e dispendiosos. Para maior rendimento desse processo evolutivo, a colaboração da Estatística no delineamento dos experimentos e avaliação de dados da pesquisa científica tem sido eficaz e dinámica.

Até a década de vinte a evolução maior tinha sido no desenvolvimento de métodos de análise das respostas obtidas com os tratamentos aplicados; desde então, começou-se a pesquisar também quais os modos mais eficientes para planejar a procura dessas respostas.

A partir de delineamentos de tratamentos mais complexos, que utilizam todas as combinações dos fatores em estudo, vārias tentativas têm sido feitas para redução do número de pontos experímentais, através de técnicas como a de confundimento e de repetição fracionada. Outra linha de pesquisa para essa redução e a dos chamados delineamentos compostos, introduzidos inicialmente na pesquisa industrial, em ensaios sequenciais, com pequeno erro experimental, e adaptados posteriormente a outros ramos da ciência. Várias modificações têm sido propostas tendo como base os 
delineamentos compostos, centrais ou não.

Desenvolveu-se, no estudo presente, a análise de um tipo de delineamento composto central, denominado Guadalupe, para très fatores, em sete níveis, através da metodologia de superfície de resposta. Foi utilizado um modelo polinomial quadrático, com polinômios ortogonais de primeiro e segundo grau, não encontrado na literatura disponível, e que elimina a correlação entre a média e os efeitos quadráticos.

E dada a sequència de operações para a realização da análise estatística, com soluções especificas para esse delineamento. Em seguida é proposta a ortogonalização para o delineamento Guadalupe; conserva-se o mesmo número total de pontos, vinte e sete, mas passa-se a ter nove níveis para cada fator, dois deles como profeção das coordenadas dos pontos fatoriais sobre os eixos. Além disso, a eficiēncia dos delineamentos Guadalupe original e Guadalupe ortogonal é calculada em relação a delineamentos de uso tradicional em pesquisa nos campos agronómico e industrial. 


\section{REVISÃO DE LITERATURA}

\subsection{Generalidades}

A idéia a respeito de funções de resposta remonta ao sëculo passado; segundo revisão apresentada por MEAD e PIKE (1975), surgiu com Fechner, em 1860, no campo de próbites.

No início do século, MITSCHERLICH (1909) aplicou-a ao estudo agronômico de respostas de produção aos níveis de fertilizantes; de acordo com HEADY e DILLON (1961), ele foi, talvez, o primeiro agrônomo a sugerir uma função não linear para relacionar o insumo nutriente à produ ção.

Sem conhecer a equação de Mitscherlich, W.J. Spillman chegou a uma função de produção exponencial, equivalente à anterior, depois de estudar resultados de experimentos de adubação com algodão, na Carolina do Norte. Suas pesquisas são relatadas por SPILLMAN e LANG (1924). Pelo trabalho de revisão citado, sabe-se que Winsor sugeriu, em 1932, o uso da curva de Gompertz para situações em que a razão do crescimento relativo decrescesse exponencialmente com o tempo; WISHART 
(1938) aplicou funções de resposta para explicar o crescimento de animais. Até FISHER (1926) não se discutiam quals os delineamentos mais apropriados para pesquisar as curvas e superfícies de resposta; a publicação de YATES (1935), com a exposição dos Experimentos Fatoriais, revelou o interesse na procura de delineamentos que possibilitem melhores respostas, com utilização simultânea de värios fatores.

Embora desenvolvidos na ärea agronómica, os experimentos fatoriais tèm sido utilizados nos mais variados campos de pesquisa. COCHRAN e COX (1957, p. 177) fizeram referència a um experimento fatorial em estudos de Neurofisiologia, com ratos. COX (1958, cap. 6 e 7 ) deu 8xemplos de aplicação em indústria têxtil, em Cièncias Biológicas, em Parasitologia e em Bioquímica, bem como em processos de produção de medicamentos. Em HICKS (1973, p. 86), exemplos em pesquisa tecnológica na indústria de computadores, de televisores, de filmes, foram dados no capítulo de ensaios fatoriais. SNEDECOR e COCHRAN (1974, p. 345) apresentaram experimentos fatoriais sobre o ganho de peso de suínos. GILL (1978, vol. 2, p. 262) relatou experimento que visa a encontrar o ponto de viscosidade mínima em leite esterilizado e concentrado, armazenado durante seis meses em temperatura ambiente.

Mas é na área agronômica que se encontra a maior utili zação dos delineamentos fatoriais.

No Brasil, värios grupos de experimentos de adubação com $N$, P e K, em diversas culturas, foram planejados como fatoriais $3 \times 3 \times 3$ ou semelhantes. STRAUSS (1951), em Pernambuco, MALAVOLTA et alii (1963) e ALVAREZ et alii (1963), em São Paulo, os ut1lizaram para a cana-de-açúcar. ARRUDA (1959) e MIRANDA (1966) relataram resultados de ensaios de milho. 
IGUE (1968) estudou o efeito de interações em cerca de cinqüenta ensalos de feijão. FUZATTO et alii (1970) analisaram ensaios fatoriais $3 \times 3 \times 3$ de algodão, selecionados entre um grupo de trezentos e setenta e sete, plantados em très anos.

Como, para mais de très fatores, os ensaios fatorials requerem nümero muito grande de unidades experimentais, FINNEY (1945) introduziu o conceito de fatoriais fracionados; são bastante üteis, princi palmente em pesquisa industrial; segundo OAVIES (1954, p. 8), o uso do fatorial completo, nesse tipo de experimentação, iria levar à avaliação de interações sem importància e a um grau de precisão desnecessário em ensaios que, pela sua natureza, já tèm um erro experimental pequeno.

BOX e WILSON (1951) desenvolveram delineamentos expertmentais para permitir encontrar o ponto de resposta máxima ou mínima, em ensaios com diferentes níveis de $k$ fatores, a partir de um nümero de observações inferior ao anteriormente preconizado. Com esse artigo foi aberta uma nova linha de procura de delineamentos que utilizaram, originalmente, cinco níveis de cada fator. Os delineamentos propostos, denominados compostos, foram introduzidos a fim de determinar condições ótimas em pesquisas químicas; mais tarde tiveram utilização em outros campos. Constam de um núcleo representado por um fatorial $2^{k}$, de um ou mais pontos centrais, e de pontos extras $-a$ e $+\alpha$ colocados em $k$ eixos, a partir do ponto central. o delineamento original de BOX e WILSON (1951), para trés fatores, tinha um único ponto central, num total de quinze pontos. No mesmo artigo, introduziram os compostos ortogonais e os compostos com variàncias iguais para os efeitos de segunda ordem. Os delineamentos compostos foram concebidos inicialmente para serem aplicados sequencialmente, primeiro utilizando os 
pontos fatorlais e depois acrescentando os demais. Este procedimento é apresentado com detalhe em DAVIES (1954, p. 541), com um exemplo seqlencial relativo a reações químicas.

Um ensaio com aplicação do composto central no campo da Zootecnia é citado em MYERS (1971, p. 132), no qual são procuradas as condiçõès ótimas de conservação do sêmen bovino para que atinja sobrevivència máxima.

BOX e HUNTER (1957) desenvolveram os delineamentos rotacionais, para $k$ fatores, com a propriedade de que é constante a varláneia da resposta estimada em pontos equidistantes do centro: como caso parti cular, apresentaram os compostos centrais rotacionais, para até oito fatores; conforme o número de fatores e de pontos centrais, eles podem, ou não, vir a ser rotacionais ortogonais. Para três fatores, os centrais rotacionais ficam com vinte pontos, constituídos de um cubo nos níveis \pm 1 , pontos axiais em $\pm 1,682$ para nível zero dos outros dois, e com seis pontos centrais (nível zero). COCHRAN e COX (1957, p. 349) deram exemplo de aná lise para um delineamento composto central rotacional com trés fatores, em nutrição vegetal.

Mas é, mais uma vez, no campo agronómico de ensaios de adubação, que se tem encontrado maior número de exemplos de aplicabilidade desse tipo de delineamentos para estudo de superfícies de resposta adaptadas através de modelos polinomiais de segundo grau. Além disso, vários autores têm tentado desenvolver, para aplicar em ensaios de adubação, extensões dos delineamentos compostos centrais, com utilização ou de maior nümero de cubos (pontos fatoriais), ou de maior número de pontos centrais, ou de maior número de pontos axiais. O uso dessas extensões, porém, não se 
restringe a esse campo.

TRAMEL (1957) sugeriu o delineamento "triplo cubo", para três fatores, com cubos de vértices nas distâncias $\pm 0,4792, \pm 0,9584$ e $\pm 1,4376$, de seis pontos axiais nas distáncias $\pm 1,9168$ e de um ponto central, num total de trinta e um pontos, com nove níveis para cada fator.

STAVROU e CADY (1967) introduziram uma modificação םo delineamento de TRAMEL (1957), colocando em dois blocos os tratamentos de cada cubo, e repetindo os pontos axiais em cada bloco. O ponto central pode ser colocado ou não; se o for, deve-se ter um em cada bloco. Com essa modificação os autores tentaram baixar o erro experimental, sem modificar a estimação dos paràmetros do modelo quadrático, que continuaram indepen dentes do efeito de blocos.

VOSS e PESEK (1967) propuseram um composto central aumentado, com dezenove pontos; os quatro tratamentos adicionados correspondem a meio cubo nos níveis $\stackrel{ \pm}{-2}$. Aplicaram o delineamento a ensaios de milho, no Estado de Iowa, em 1959; em 1960 conduziram novos ensaios com acréscimo de mais quatro tratamentos, para remover o efeito do confundimento existente. O delineamento ficou, então, com vinte e três pontos: dois cubos, um nos níveis \pm , outro em $\stackrel{ \pm}{-2}$; seis axiais nos níveis $\stackrel{ \pm}{-2}$ de cada fator, para níveis médios dos outros dois fatores, e um ponto central.

Surgiram algumas modificações desse delineamento, ainda com vinte e três pontos; para sete níveis, VANDERLIP e PESEK (1970) construíram o delineamento com um cubo nos níveis \pm-3 , outro nos níveis $\pm_{-1}$, pontos axiais em $\stackrel{+}{-2}$, para níveis médios dos outros fatores, e um ponto central no nível zero.

CHRISTIANS et alii (1979) colocaram seis repetições no 
ponto central do delineamento duplo cubo, ficando com cubos nos níveis \pm , $\stackrel{ \pm}{-2}$, seis pontos axiais nos níveis $\stackrel{+}{-3}$, num total de vinte e oito pontos experimentais.

Foi proposto no México o delineamento "San Cristóbal". E um composto modificado, desenvolvido por ROJAS (1963, pp. 197 a 203) e, segundo ele, com a finalidade de incluir um tratamento testemunha. Para $k$ fatores, consta dos vértices de um hipercubo nos níveis $\stackrel{+}{-1}$; de um trata mento no centro do cubo (nível zero), e $k$ pontos em semi-eixos que partem do centro, cada um com nivel 2 de um dos fatores e nivel zero dos restan tes. Exemplo de sua aplicação encontra-se em MARTINEZ GARZA (1978, p. 101). Posteriormente ROJAS (1972, pp. 1085-1093) propōs o "San Cristóbal ortogonalizado"; constitui, como o original, uma família de delineamentos para qualquer número de fatores. Consta de:

a) um número a de repetições de um fatorial $2^{k}$ nos níveis -1 e +1 de

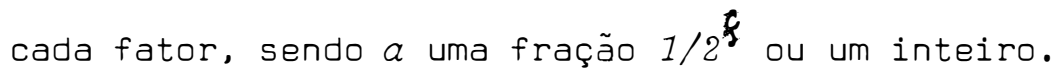

b) um nümero $m$ de repetições do centro do hipercubo, no nível zero de cada fator.

c) pontos axiais, correspondentes a $e$ pontos no nível - $\alpha$ e de um ponto no nível ca de cada fator, para nível zero dos restantes, com a restrição $0<a \leq 1$.

0 número total de tratamentos diferentes e $2^{k}+2 k+1$; o nümero de unidades experimentais, por repetição básica, é $n=a 2^{k}+k(c+1)+m$. DÃVILA (1979, pp. 131 a 156) deu características e grä ficos de uma série de delineamentos usados em exoerimentos com fertilizantes. Para dois fatores, mencionou os publicados em revista do CIMMYT, designados "diamante duplo" (um fatorial $3 \times 3 \mathrm{com}$ vértices em $\stackrel{+}{-1} e$ quatro 
axiais com $\alpha= \pm 1 / 2$; "quadrado duplo" (um fatorial $3 \times 3$ com vértices em $\pm_{1}$ e um quadrado com pontos nas diagonais do fatorial); e "quadrado triplo" (um fatorial $3 \times 3$ em $\pm_{1}$ e dois quadrados com vértices nas diagonais do fatorial). Entre os de trés fatores, além dos já mencionados San Cristóbal, composto central rotacional e triplo cubo, citou a utilização do "monocubo", delineamento com quatorze tratamentos, em programa de adubação no México.

No Brasil, uma primeira tentativa para modificação do composto central original, de que se tem conhecimento, foi a proposta em reunião da Região Brasileira da Sociedade Internacional de Biometria, em $1966\left(^{1}\right)$. As características do delineamento estão especificadas em CONAGIN et alii (1969, pp. 183 a 201); desenvolvido inicialmente para trés fatores, consta de dois cubos nos níveis $\stackrel{+}{-1} e^{ \pm} \stackrel{+}{-2}$; de um ponto central; e de doze pontos axiais nos níveis $\stackrel{+}{-1} e^{ \pm}-2$ de cada fator, para nível médio dos outros dois, num total de vinte e nove pontos.

BATISTA (1978) apresentou um novo tipo de delineamento, denominado "em círculos", que teve por base, na formulação, os princípios de utilização de maior número de níveis para cada fator, menor nümero de combinações entre eles e independència na estimação dos coeficientes. Consta de dois conjuntos de oito pontos às distäncias $\sqrt{2}$ e $\alpha \sqrt{2}$ do centro, e mais $P$ pontos centrais, que abrangem nove níveis diferentes de cada fator. Os delineamentos compostos permitem o estudo de superfície de resposta com adaptação de modelos polinomiais de segundo grau, em $x$ ou em $\sqrt{x}$. As respostas levarão a conclusões satisfatörias se houver, den-

(1) Delineamento experimental duplo cubo com pontos intermediärios sobre os trés eixos. Mencionado em 1967, Biometrics 23: p. 879. 
tro dos níveis pesquisados no ensaio, um ponto de máximo ou de mínimo, conforme a natureza do problema estudado. PIMENTEL GOMES (1969) assinalou a necessidade da caracterização desses pontos críticos, mencionando a existência de vários trabalhos com conclusōes erradas por não tomarem esse cuidado; lembrou, ainda, que esse ponto crítico deve ser buscado dentro da região estudada pelo ensaio.

Na comparação entre delineamentos experimentais utilizados para obtenção de superfícies de resposta polinomiais de segundo grau, BOX e WILSON (1951) levaram em conta a precisão na estimativa dos parâme tros da função e o vício que pode surgir pela não adequação do modelo.

Considerando somente o aspecto da precisão, a comparação é feita entre as matrizes de dispersão; deve-se antes ajustä-las para o nümero de pontos $e$, se os níveis dos fatores de cada delineamento estiverem em bases diferentes de escala, eles devem ser trazidos para uma escala comum, e as conclusões vão depender da definição dessa medida. Os autores sugeriram, no artigo, que dois delineamentos a serem comparados tenham iguais os segundos momentos em relação à média; consideraram razoäveis os resultados obtidos até então por esse critério, mas que prssivelmente poderia haver outras medidas mais apropriadas em certos casos.

PIMENTEL GOMES e CAMPOS (1972) propõem como melhor, em experimentos com fertilizantes, que se tenha, para escala comum, a mesma amplitude entre os níveis extremos de cada fator.

\subsection{O Delineamento Guadalupe}

Pouco se sabe a respeito de como surgiu o delineamento 
Guadalupe; tem-se a citação, em PAEZ e SILVA (1975), de ter sido apresen tado em curso de pós-graduação em Turrialba, Costa Rica, em 1972.

Os autores mencionam que se está generalizando, em cer tas estações experimentais da Europa e da América Central, a tendéncia no sentido do uso de maior nümero de níveis dos fatores, para estimar a função de produção. E é dito que o Guadalupe foi desenvolvido, em parte, para satisfazer a pesquisadores da escola que preconiza: "usar maior número de níveis para descrever a superfície de resposta biológica é mais seguro que usar poucos níveis e muitas repetiçöes espaciais".

Em seguida, PAEZ e SILVA (1975) definem os tratamentos do delineamento, chamando-os de radiais, centrais e polares. No presente trabalho os tratamentos serão chamados, respectivamente, de axiais, centrais e fatoriais, correspondentes aos pontos sobre os eixos, aos centrais e aos vértices do hipercubo. .

O total de tratamentos para $k$ fatores em sete níveis é igual a $2^{k-f}+6 k+c$, onde $6 k$ é o número de tratamentos radiais, $c$ repre. senta o nümero de repetições do tratamento central e $2^{k-f}$ representa a parte fatorial, que pode ser uma repetição fracionada ou o fatorial completo, dependendo de $k$. Para $k$ igual a dois, trés ou quatro, $f$ é geral mente igual a zero; se o número de fatores é maior que quatro, $f=1$; à me dida que $k$ cresce, f poderá crescer para reduzir o número de tratamentos fatoriais, através da técnica do fracionamento.

São sugeridos por Páez e Silva dois modelos: um de apreciação rápida e sem cálculos matemáticos, ajustando duas retas à parte axial e central, e determinando o ponto de intersecção das retas, para cada um dos fatores; o outro se refere a uma superfície de segundo grau, uti- 
lizàndo todos os tratamentos.

Em comunicação pessoal Páez sugeriu a realização da anālise da superfície de resposta através de método padrão de regressão múltipla, usando modelo quadrático com dez parāmetros; indicou a matriz das variāveis independentes, com níveis já codificados. A matriz $X^{\prime} X$ obtida mostra que somente os efeitos lineares e as interações vão ser independentes, e a média e os efeitos quadráticos irão apresentar correlações. 
15.

\section{METODOLOGIA}

\subsection{Características do delineamento Guadalupe para très fatores}

Para $k=3$, o delineamento Guadalupe permite estudar

três fatores em sete níveis. Na expressão geral $2^{k-f}+6 k+c, f$ é igual a zero, pois, para $k=3$, o número 8 de pontos fatoriais é bastante razoāvel. Fica-se, ainda, com $6 k=18$ tratamentos axiais, $c=1$ tratamento central, num total $N$ de 27 pontos. O conjunto de $N$ pontos constitui o "delineamento de tratamentos".

E dada a seguir a composição dos tratamentos, onde os fatores serão indicados por $i, j, k$ e os níveis vão de zero a seis, independentemente das unidades originais de medida de cada fator.

Pontos Fatoriais

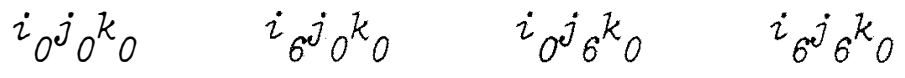

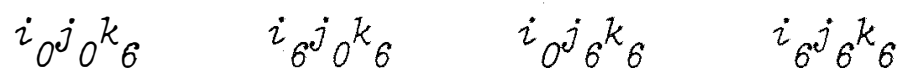

$$
\begin{aligned}
& \text { Ponto Central } \\
& i_{3} j_{3} k_{3}
\end{aligned}
$$


Pontos Axiais

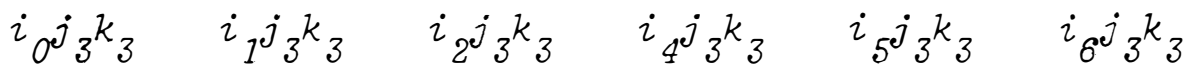

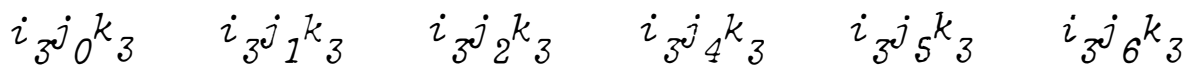

$$
\begin{aligned}
& i_{3^{j}} 3^{k_{0}} \quad i_{3^{j}}{ }^{k_{1}} \quad i_{3^{j}} 3_{2}^{k_{2}} \quad i_{3^{j}}{ }^{k_{4}} \quad i_{3^{j}} 3_{5}^{k_{5}} \quad i_{3^{j}}{ }^{k_{6}}
\end{aligned}
$$

Pode-se notar que os pontos fatoriais formam um cubo que

limita a região experimental, com os axiais extremos pertencentes às faces do cubo.

$$
\text { As coordenadas do conjunto de pontos que define o deli- }
$$

neamento podem ser colocadas em forma de matriz, constituída por 27 linhas e 3 colunas. Em cada linha da matriz D, chamada "matriz do delineamento", estão representados os níveis $x$ dos fatores $i, j, k$ aplicados a uma unidade experimental.

$$
D=\left[\begin{array}{lll}
i & j & k \\
0 & 0 & 0 \\
6 & 0 & 0 \\
0 & 6 & 0 \\
6 & 6 & 0 \\
0 & 0 & 6 \\
6 & 0 & 6 \\
0 & 6 & 6 \\
6 & 6 & 6 \\
3 & 3 & 3 \\
0 & 3 & 3 \\
1 & 3 & 3 \\
2 & 3 & 3 \\
4 & 3 & 3 \\
5 & 3 & 3 \\
6 & 3 & 3 \\
3 & 0 & 3 \\
3 & 1 & 3 \\
3 & 2 & 3 \\
3 & 4 & 3 \\
3 & 5 & 3 \\
3 & 6 & 3 \\
3 & 3 & 0 \\
3 & 3 & 1 \\
3 & 3 & 2 \\
3 & 3 & 4 \\
3 & 3 & 5 \\
3 & 3 & 6 \\
& & \\
& & \\
3 \\
3
\end{array}\right)
$$




\subsection{Metodologia de superfície de resposta}

A anālise do delineamento Guadalupe foi feita segundo a metodologia de superfície de resposta polinomial.

Levando em conta o fato de o delineamento Guadalupe ser um tipo de composto central, criado para permitir adaptação de funções de segundo grau, o modelo escolnido para relacionar as respostas (variável y) com os níveis dos fatores (variável $x$ ) foi uma função de resposta polinomial quadrática.

$$
\begin{aligned}
& \text { Poder-se-ia ter usado o modelo comumente sugerido } \\
& \hat{y}=b_{0}+\sum_{i=1}^{3} b_{i} x_{i}+{ }_{i=1}^{3} b_{i i} x_{i}^{2}+\sum_{i}, \sum_{j} b_{i j} x_{i} x_{j} \\
& i<j
\end{aligned}
$$

onde $x_{i}$ é a variável reduzida ou codificada $x_{i}=\left(X_{i}-\bar{X}_{i}\right) / a_{i}$.

Pelas características do delineamento, com esse modelo os parāmetros correspondentes aos efeitos quadráticos puros não ficariam independentes da média, sendo necessária nova parametrização para que a independència acontecesse (DAVIES, 1954, p. 535).

Procurando deixar o efeito médio sempre independente, foram usados polinômios $p_{1}$ e $p_{2}$, de $1^{\circ}$ e de $2^{\circ}$ grau, correspondentes aos componentes linear e quadrático, ortogonais entre si e também ao polinômio $P_{0}$, relacionado com a média e tomado sempre como $P_{0}=1$. Então, as condições de ortogonalidade entre a média e os efeitos lineares e quadräticos dos fatores se resumem nas condições de ortogonalidade entre os polinômios $p_{1}$ e $p_{2}$.

$$
\text { Usou-se o método geral de ortogonalização dos polinó- }
$$
mios, dado de modo bastante simples em GRANDAGE (1958) e, recentemente, 
18.

em NOGUEIRA (1979). Embora para níveis equidistantes seja mais fácil a reparametrização, o uso dos polinômios ortogonais padroniza a sequência de operações e uniformiza o método para encontro do valor das variáveis para qualquer modelo.

4.3. Modelo de regressão linear: cálculo das estimativas dos parâmetros e de suas variâncias e covariâncias; anālise da variância e testes de hipötese; intervalos de confiança

A função de resposta, suposta polinomial e adequada, foi expressa segundo o modelo de regressão linear em $q=10$ parámetros $b$, para três fatores $i, j$ e $k$, cujos $v$ níveis equidistantes variam de zero a seis. A resposta em cada um dos $N$ pontos do delineamento é dada por:

$$
\begin{aligned}
& y_{i j k}=b_{0} p_{0}+b_{1 i} p_{1 i}+b_{1 j} p_{1 j}+b_{1 k} p_{1 k}+b_{2 i} p_{2 i}+b_{2 j} p_{2 j}+b_{2 k} p_{2 k}+
\end{aligned}
$$

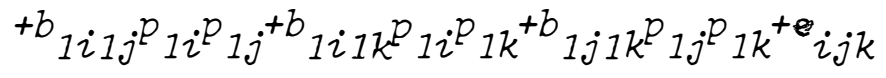

No modelo, y é uma variável aleatória dependente de $b$ e de $p$; os parâmetros $b$ são desconhecidos, a serem estimados; $P_{1} e$ $P_{2}$ são polinômios ortogonais de $1^{8}$ e de $2^{8}$ grau, respectivamente; $e$ ë uma variável aleatória normalmente distribuída, com média zero e variància $\sigma^{2}$, isto é: eqN(O; $\left.\sigma^{2}\right)$.

Visto que cada um dos fatores $i, j, k$ entra, na compo sição dos tratamentos, com igual nümero de níveis, eles são chamados genericamente de fator $m$ quando se calculam os polinômios $p_{1 m}$ e $P_{2 m}$. Todo o desenvolvimento metodológico do modelo de regressão linear (nos parâmetros b) estä baseado em definições, fórmulas dedu- 
zidas e conceitos emitidos nos livros de ANOERSON e BANCROFT (1952, cap. 14), GRAYBILL (1961, cap. 6), SEARLE (1971, cap. 3), no artigo de BOX e WILSON (1951) e em HOFFMAN e VIEIRA (1978, cap. 4). 0 modelo apresentado pode ser dado, em forma matricial, como:

$$
Y=P \beta+\varepsilon \quad
$$

Y é o vetor coluna, com 27 linhas, das variáveis aleátórias $y_{i j k}$,que são função dos níveis $x$ através dos polinômios $p_{1}$ e $p_{2}$

$\beta$ é o vetor coluna dos dez paràmetros $b$ e $\varepsilon$ o vetor coluna dos erros $e_{i j k}$, com 27 linhas; $\varepsilon \sim N\left(0 ; I \sigma^{2}\right)$.

$P$ é a "matriz das variáveis independentes", construída a partir da matriz $D(27 \times 3)$, do delineamento, e das funções que definem os polinômios ortogonais, com 27 linhas e 10 colunas.

0 vetor $B$, estimado pelo método dos quadrados mínimos, apresenta a solução única

$$
\hat{B}=\left(P^{\prime} P\right)^{-1} P^{\prime} Y
$$

desde que $\left(P^{\prime} P\right)$ seja não-singular, o que acontece se a característica da matriz $P$ for igual ao número de paràmetros.

Chamando de $S$ a matriz $\left(P^{\prime} P\right)$,

$$
\hat{B}=S^{-1} P^{\prime} Y \text {. }
$$

A "matriz de variàncias e covariàncias de $\vec{B}$ ", ou "matriz de dispersão de $\widehat{\beta} "$, é dada por:

$$
\operatorname{Cov} \hat{B}=\left(P^{\prime} P\right)^{-1} \sigma^{2}=S^{-1} \sigma^{2} .
$$

A expressão mostra que as variâncias e covariàncias das estimativas dos paràmetros dependem do delineamento experimental e da fun- 
ção de resposta utilizada.

$$
\begin{aligned}
& \text { Sendo } \vec{B}=S^{-1} P^{\prime} Y \text { e } E(\hat{B})=B \text {, tem-se que } \\
& \qquad \widehat{B} \sim N\left(B ; S^{-1} \sigma^{2}\right) . \\
& \text { Geralmente se considera estimativa não-viciada de } \sigma^{2} \text { a }
\end{aligned}
$$

expressão

$$
s^{2}=\frac{1}{N-q}\left\{Y^{\prime} Y-\hat{B}^{\prime} P^{\prime} Y\right\},
$$

que corresponde ao quadrado médio do erro, uma vez que $Y^{\prime} Y$ é a soma de quadrados total (SQ Total) e $\hat{B}^{\prime} P^{\prime} Y$ é a redução, na soma de quadrados, devida à adaptação do modelo de regressão linear ( $S Q$ Reg.).

$$
\text { Com os elementos já mencionados, pode-se construir o }
$$
quadro da análise da variància e realizar um teste global para os parâme-

\begin{tabular}{|c|c|c|c|}
\hline$F . V$. & S.Q. & G.L. & Q.M. \\
\hline Regressão $\quad(q=10)$ & $\hat{B^{\prime}} P^{\prime} Y$ & $(10)$ & \\
\hline Média & $b_{0} A$ & 1 & \\
\hline Regressão $(q-1)$ & $\hat{b}_{1 i^{B+b}}{ }_{1 j} C+\ldots+\hat{b}_{1 j 1 k^{J}}$ & 9 & \\
\hline Erro & $Y^{\prime} Y-\hat{B}^{\prime} P^{\prime} Y$ & $\cdot 17$ & $s^{2}$ \\
\hline Total & $Y^{\prime} y$ & 27 & \\
\hline
\end{tabular}
tros do modelo. Quando há componentes ortogonais a SQ Regressão fica subdividida em parcelas correspondentes; os testes $F$ são feitos com os graus de liberdade respectivos. No modelo, a média é sempre ortogonal aos outros componentes.

Se se quiser testar se um parāmetro genérico $b$ (exceto o correspondente à média e os ortogonais a todos os restantes) pode ser 
considerado igual a zero, usa-se o método do resíduo condicional, devido a Fisher, e bastante detalhado em GRAYBILL (1961, pp. 133-140).

0 vetor das estimativas dos valores esperados de $y_{i j k}$, indicado por $\mathcal{Y}$, é dado por:

$$
\hat{Y}=P \hat{B} \quad \text {. }
$$

Chamando de $P_{i j k}^{\prime} \circ$ vetor linha com 10 colunas, cujos elementos correspondem à linha da matriz $P$ relativa a uma determinada combinação dos fatores $i, j, k$,

$$
\hat{y}_{i j k}=P_{i j k}^{\prime} \hat{B}
$$

e a variância de cada $\hat{y}_{i j k}$ é da forma

$$
\operatorname{Var}\left(\hat{y}_{i, j k}\right)=P_{i, j k}^{\prime} S^{-1} P_{i, j k} \sigma^{2}
$$

Foi visto que $\hat{B} \sim N\left(\beta ; S^{-1} \sigma^{2}\right)$; logo, cada um dos $\widehat{b} \sim N\left(b ; d \sigma^{2}\right)$, onde $d$ designa o elemento da diagonal de $S^{-1}$ correspondente ao parâmetro genérico $b$. Sabe-se, também, que $\vec{B} \& s^{2}$ são independentes e, em consequéncia, $\hat{B}$ e $(N-q) s^{2} / \sigma^{2}$ também o são. Portanto, havendo interesse, o intervalo de confiança de $b$, com coeficiente de confiança $1-\alpha$, e $t$ com $N-q$ graus de liberdade, pode ser calculado como segue:

$$
\begin{aligned}
& b-t_{\alpha / 2} s \sqrt{d} \leq b \leq b+t_{\alpha / 2} s \sqrt{d} \\
& \text { Como } \hat{y}_{i j k} \sim N\left(P_{i j k}^{\prime} \beta ; P_{i, j k}^{\prime} S^{-1} P_{i j k} \sigma^{2}\right), \quad 0 \text { intervalo de }
\end{aligned}
$$
confiança de $y_{i . j k}$, com coeficiente de confiança $1-\alpha$, e $t$ com $N-q$ graus de liberdade, pode ser calculado por:

$$
\hat{y}_{i j k}{ }_{\alpha / 2} \Delta \sqrt{P_{i j k}^{\prime} S^{-1} P_{i j k}} \leq y_{i j k} \leq \vec{y}_{i j k}+t_{\alpha / 2} s \sqrt{P_{i j k}^{\prime} S^{-1} P_{i j k}} \text {. }
$$


22.

\subsection{Aplicação do modelo de regressão linear polinomial quadrätica ao delineamento Guadalupe com très fatores}

No modelo de regressão linear polinomial em dez parâmetros, expresso no item anterior, os polinómios $p_{1 m}$ e $p_{2 m}$, de primeiro e de segundo grau, são da forma geral

$$
p_{1 m}=\alpha_{1}+x_{m} \quad \text { e } \quad p_{2 m}=\alpha_{2}+\gamma_{2} x_{m}+x_{m}^{2}
$$

A partir das coordenadas $x$ dos pontos do delineamento, dadas na matriz $D$, tem-se a expressão dos polinōmios $P_{1 m}$ e $P_{2 m}$ para os 27 pontos.

Impostas as condições de ortogonalidade entre os vetores

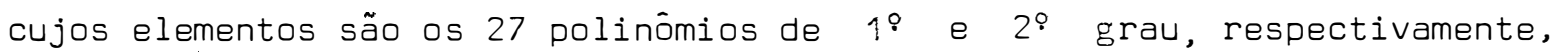
$\left(\sum p_{1 m}=0 ; \sum p_{2 m}=0 ; \sum p_{1 m} p_{2 m}=0\right)$ chega-se ao sistema

$$
\left\{\begin{aligned}
27 \alpha_{1}+81 & =0 \\
27 \alpha_{2}+81 \gamma_{2}+343 & =0 \\
100 \gamma_{2}+600 & =0
\end{aligned}\right.
$$

cuja solução resulta nas expresșões

$$
\begin{aligned}
& p_{1 m}=-3+x_{m}, \quad p_{2 m}=5,29629-6 \dot{x}_{m}+x_{m}^{2} . \\
& \text { Obtidos } p_{1 m} \text { e } p_{2 m} \text {, segue-se imediatamente a cons- }
\end{aligned}
$$

trução de $P$, matriz das variáveis independentes; para melhor ajuste das condições de ortogonalidade, os valores positivos encontrados para $p_{2 m}$ foram aproximados até a quarta casa decimal. 


\begin{tabular}{|c|c|c|c|c|c|c|c|c|c|c|}
\hline $\begin{array}{l}\text { Coor. } \\
\text { Pont. }\end{array}$ & $p_{0}$ & $p_{1 i}$ & $p_{1 j}$ & $p_{1 k}$ & $p_{2 i}$ & $P_{2 j}$ & $P_{2 k}$ & $P_{1 i 1 j}$ & $P_{1 i 1 k}$ & $P_{1 j 1 k}$ \\
\hline 000 & 1 & -3 & -3 & -3 & 5,29630 & 5,29630 & 5,29630 & 9 & 9 & 9 \\
\hline 600 & 1 & 3 & -3 & -3 & 5,29630 & 5,29630 & 5,29630 & -9 & -9 & 9 \\
\hline 060 & 1 & -3 & 3 & -3 & 5,29630 & 5,29630 & 5,29630 & -9 & 9 & -9 \\
\hline 660 & 1 & 3 & 3 & -3 & 5,29630 & 5,29630 & 5,29630 & 9 & -9 & -9 \\
\hline 006 & 1 & -3 & -3 & 3 & 5,29630 & 5,29630 & 5,29630 & 9 & -9 & -9 \\
\hline 606 & 1 & 3 & -3 & 3 & 5,29630 & 5,29630 & 5,29630 & -9 & 9 & -9 \\
\hline 066 & 1 & -3 & 3 & 3 & 5,29630 & 5,29630 & 5,29630 & -9 & -9 & 9 \\
\hline 666 & 1 & 3 & 3 & 3 & 5,29630 & 5,29630 & 5,29630 & 9 & 9 & 9 \\
\hline 033 & 1 & -3 & 0 & 0 & 5,29630 & $-3,70371$ & $-3,70371$ & 0 & 0 & 0 \\
\hline 133 & 1 & -2 & 0 & 0 & 0,29630 & $-3,70371$ & $-3,70371$ & 0 & 0 & 0 \\
\hline 233 & 1 & -1 & 0 & 0 & $-2,70371$ & $-3,70371$ & $-3,70371$ & 0 & 0 & 0 \\
\hline 433 & 1 & 1 & a & 0 & $-2,70371$ & $-3,70371$ & $-3,70371$ & 0 & 0 & 0 \\
\hline $533 \quad P=$ & 1 & 2 & 0 & 0 & 0,29630 & $-3,70371$ & $-3,70371$ & 0 & 0 & 0 \\
\hline 633 & 1 & 3 & 0 & 0 & 5,29630 & $-3,70371$ & $-3,70371$ & 0 & 0 & 0 \\
\hline 303 & 1 & 0 & -3 & 0 & $-3,70371$ & 5,29630 & $-3,70371$ & 0 & 0 & 0 \\
\hline 313 & 1 & 0 & -2 & 0 & $-3,70371$ & 0,29630 & $-3,70371$ & 0 & 0 & 0 \\
\hline 323 & 1 & 0 & -1 & 0 & $-3,70371$ & $-2,70371$ & $-3,70371$ & 0 & 0 & 0 \\
\hline 343 & 1 & 0 & 1 & 0 & $-3,70371$ & $-2,70371$ & $-3,70371$ & 0 & 0 & 0 \\
\hline 353 & 1 & 0 & 2 & 0 & $-3,70371$ & 0,29630 & $-3,70371$ & 0 & 0 & 0 \\
\hline 363 & 1 & 0 & 3 & 0 & $-3,70371$ & 5,29630 & $-3,70371$ & 0 & 0 & 0 \\
\hline 330 & 1 & 0 & 0 & -3 & $-3,70371$ & $-3,70371$ & 5,29630 & 0 & 0 & 0 \\
\hline 331 & 1 & 0 & 0 & -2 & $-3,70371$ & $-3,70371$ & 0,29630 & 0 & 0 & 0 \\
\hline 332 & 1 & 0 & 0 & -1 & $-3,70371$ & $-3,70371$ & $-2,70371$ & 0 & 0 & 0 \\
\hline 334 & 1 & 0 & 0 & 1 & $-3,70371$ & $-3,70371$ & $-2,70371$ & 0 & 0 & 0 \\
\hline 335 & 1 & 0 & 0 & 2 & $-3,70371$ & $-3,70371$ & 0,29630 & 0 & 0 & 0 \\
\hline 336 & 1 & 0 & 0 & 3 & $-3,70371$ & $-3,70371$ & 5,29630 & 0 & 0 & 0 \\
\hline 333 & 1 & 0 & 0 & 0 & $-3,70371$ & $-3,70371$ & $-3,70371$ & 0 & 0 & 0 \\
\hline
\end{tabular}

As linhas da matriz $P$ são indicadas pelo vetor $P_{i j k}^{\prime}$, com dez colunas; as colunas da matriz, pelo vetor indicado genericamente por $P_{b}$, constituído de 27 linhas.

$$
\text { Com } P \text { se obtêm } S=\left(P^{\prime} P\right) \text { e } S^{-1} \text {, transcritas a se- }
$$

guir. 

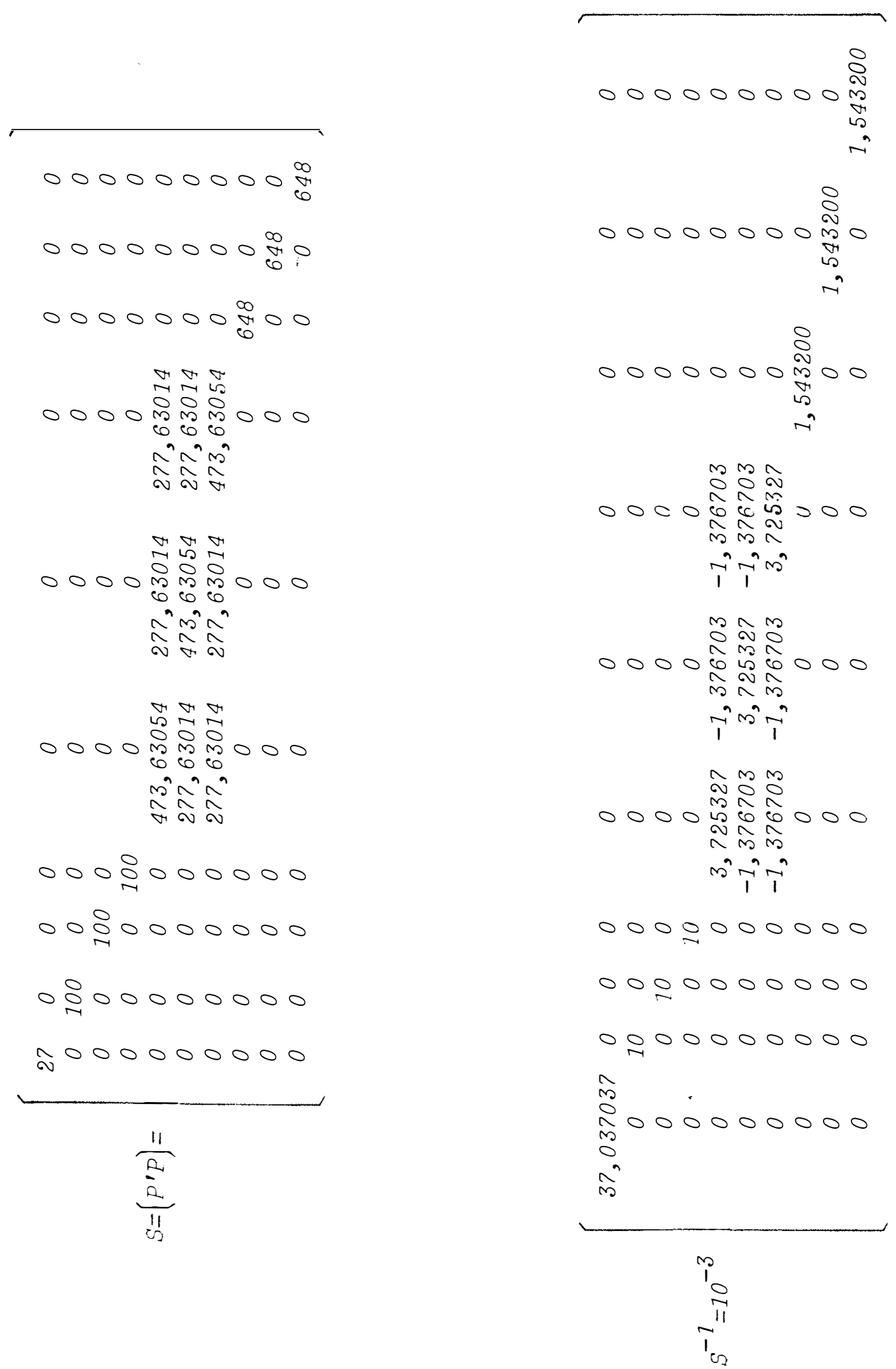
Para a estimação do vetor $\beta$ deve-se construir ainda - vetor coluna $P^{\prime} Y$, com dez elementos $A, B, \ldots, J$ que têm as expressões que se seguem:

$$
\begin{aligned}
& A=P_{b_{0}^{\prime}}^{Y}=\sum Y_{i j k} \\
& B=P_{b}^{\prime} \underset{1 i}{Y} \quad E=P_{b_{2 i}^{\prime}}^{Y} \quad I=P_{b_{1 i 1 j}^{\prime}}^{Y} \\
& c=P_{b_{1 j}^{\prime}}^{Y} \quad F=P_{b_{2 j}^{\prime}}^{Y} \quad J=P_{b_{1 i 1 k}^{\prime}}^{Y} \\
& D=P_{b_{1 k}^{\prime}}^{Y} \quad G=P_{b_{2 k}^{\prime}}^{Y} \quad K=P_{b_{1 j 1 k}^{\prime}}^{Y} \text {. }
\end{aligned}
$$

$\widehat{B}=S^{-1} P^{\prime} Y$ tem como estimativas:

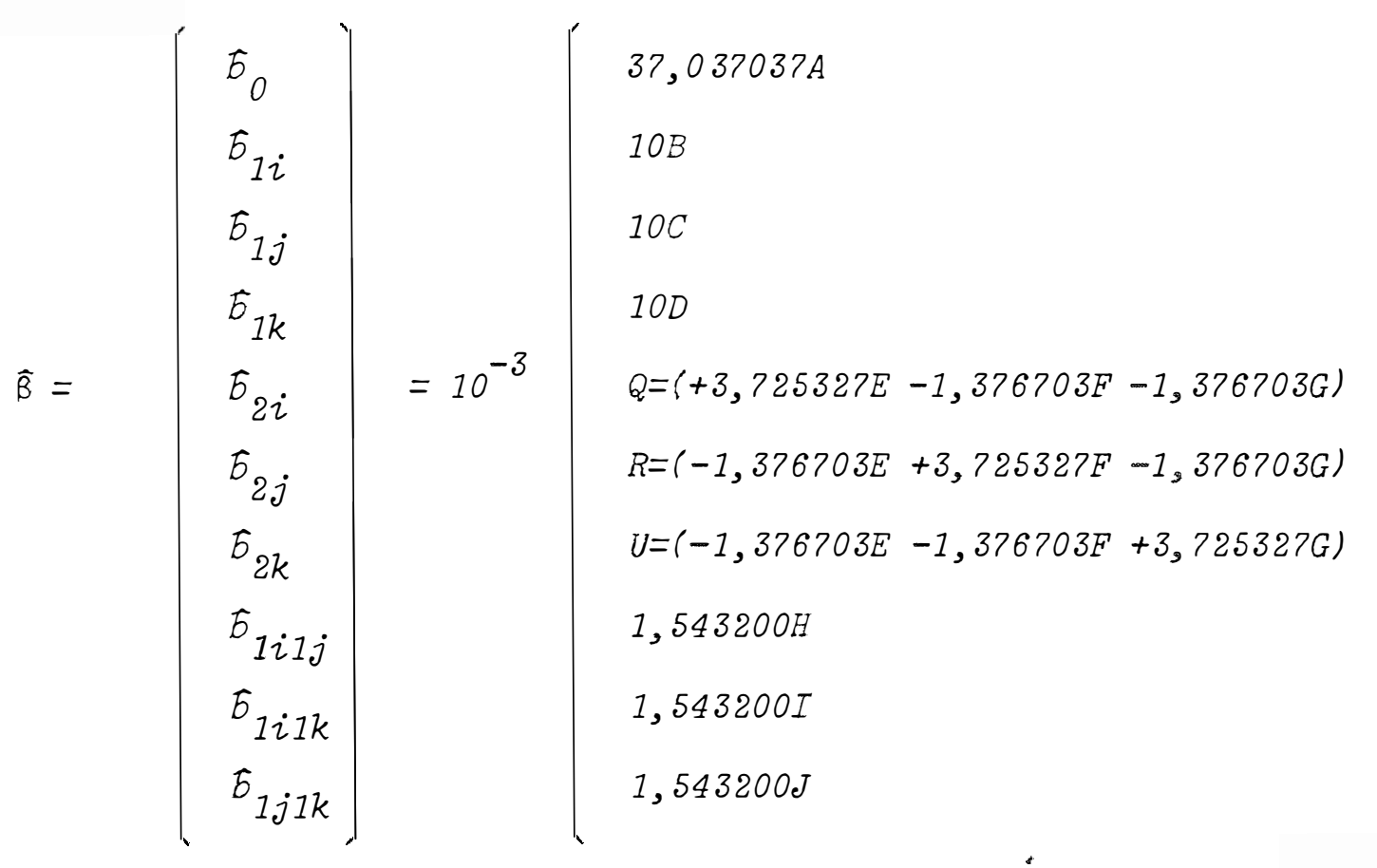

As variáncias e covariàncias dos elementos de $\hat{\beta}$, que se encontram, respectivamente, sobre e fora da diagonal de $S^{-1} \sigma^{2}$, vêm em seguida: 
26.

$$
\begin{aligned}
& \operatorname{Var} b_{0} \quad=0,037037 \sigma^{2} \\
& \operatorname{Var} \hat{b}_{1 i}=\operatorname{var} \hat{b}_{1 j}=\operatorname{var} b_{1 k}=0,010000 \sigma^{2} \\
& \operatorname{var} \hat{b}_{2 i}=\operatorname{var} \hat{b}_{2 j}=\operatorname{var} \hat{b}_{2 k}=0,003725 \sigma^{2} \\
& \operatorname{Var} \hat{b}_{1 i 1 j}=\operatorname{var} b_{1 i 1 k}=\operatorname{var} \hat{b}_{1 j 1 k}=0,001543 \sigma^{2} \\
& \operatorname{Cov}\left(\hat{b}_{2 i}, \hat{b}_{2 j}\right)=\operatorname{Cov}\left(\hat{b}_{2 i}, \hat{b}_{2 k}\right)=\operatorname{Cov}\left(\hat{b}_{2 j}, \hat{b}_{2 k}\right)=-0,001376 \sigma^{2} .
\end{aligned}
$$

A variāncia dos valores estimados tem por expressão:

$$
\operatorname{Var}\left(\hat{y}_{i \cdot j k}\right)=\left\{\begin{array}{l}
0,037037 p_{0}^{2}+0,010000\left(p_{1 i}^{2}+p_{1 j}^{2}+p_{1 k}^{2}\right)+ \\
+0,003725\left(p_{2 i}^{2}+p_{2 j}^{2}+p_{2 k}^{2}\right)+ \\
+0,001543\left(\left(p_{1 i} p_{1 j}\right)^{2}+\left(p_{1 i} p_{1 k}\right)^{2}+\left(p_{1 j} p_{1 k}\right)^{2}\right)- \\
-0,002753\left(\left(p_{2 i} p_{2 j}+p_{2 i} p_{2 k}+p_{2 j} p_{2 k}\right)\right)
\end{array}\right\} \sigma^{2}
$$

Realizados os cälculos, as variâncias são as seguintes:

a) pontos fatoriais : $0,7638 \sigma^{2}$

b) ponto central: $0,0770 \sigma^{2}$

c) pontos axiais simétricos, a partir dos extremos para o centro do delineamento : 0,4040 $\sigma^{2}, 0,1478 \sigma^{2}$ e $0,0836 \sigma^{2}$.

A disposição dos elementos da matriz $S^{-1}$ mostra quais são os componentes ortogonais na soma de quadrados da regressão, dada por $\hat{\beta}^{\prime} P^{\prime} Y$. O quadro da análise da variância apresenta a decomposição dessa soma de quadrados, no caso de uma só repetição.

Por esse quadro, dado na próxima pägina, vê-se que os componentes quadräticos puros são correlacionados.

Os componentes lineares e os correspondentes às interações são testacos individualmente, tomando-se $F$ com 1 e 17 graus de liberdade. 


\begin{tabular}{|c|c|c|c|}
\hline$F \cdot V$. & $S \cdot Q$. & G.L. & Q.M. \\
\hline Regressão & $\hat{B}^{\prime} P^{\prime} Y$ & $(10)$ & \\
\hline${ }^{b} \sigma^{A}$ (média) & $0,037037 A^{2}$ & 1 & $0,037037 A^{2}$ \\
\hline$b_{1 i^{B}}$ & $0,010000 B^{2}$ & 1 & $0,010000 B^{2}$ \\
\hline$b_{1 j} C$ & $0,010000 c^{2}$ & 1 & $0,010000 c^{2}$ \\
\hline$b_{1 k} D$ & $0,010000 D^{2}$ & 1 & $0,010000 D^{2}$ \\
\hline$\widehat{b}_{2 i} E+\widehat{b}_{2 j} F+\widehat{b}_{2 k} G$ & $10^{-3}(Q E+R F+U G)$ & 3 & $10^{-3}(Q E+R F+U G) \div 3$ \\
\hline$b_{1 i 1 j^{H}}$ & $0,001543 H^{2}$ & 1 & $0,001543 H^{2}$ \\
\hline$b_{1 i 1 k}$ & $0,001543 I^{2}$ & 1 & $0,001543 I^{2}$ \\
\hline$b_{1 j 1 k^{J}}$ & $0,001543 J^{2}$ & 1 & $0,001543 J^{2}$ \\
\hline Erro & $Y^{\prime} Y-\hat{B}^{\prime} P^{\prime} Y$ & 17 & $\left(Y^{\prime} Y-\hat{B}^{\prime} P^{\prime} Y\right) \div 17$ \\
\hline Total & $Y^{\prime} Y$ & 27 & \\
\hline
\end{tabular}

Como os componentes quadräticos puros são de grande interesse na caracterização da superfície de resposta e na anālise económica dos resultados, usa-se o método do resíduo condicional para testä-los individualmente.

E dada a inversa da matriz $S_{q}(2 \times 2)$, necessäria para o cálculo da soma de quadrados, correspondente a cada componente quadrätico, ajustada para os outros dois.

$$
\left.S_{q}^{-1}=10^{-3} \mid \begin{array}{cc}
3,216563 & -1,885467 \\
-1,885467 & 3,216563
\end{array}\right]
$$


28.

\title{
5. ORTOGONALIZAÇÃO DO DELINEAMENTO GUADALUPE COM TRES FATORES
}

\subsection{Generalidades}

\begin{abstract}
O delineamento Guadalupe, conforme foi proposto, tem os componentes quadräticos puros não independentes e a grandeza entre as covariâncias existentes representa cerca de um terço da grandeza de suas variāncias.
\end{abstract}

A fim de permitir a estimação independente de todos os componentes do modelo polinomial quadrático é realizada a ortogonalização da matriz $S$. Para isso devem-se anular os elementos de $S$ relativos aos produtos dos vetores correspondentes a $p_{2 i} p_{2 j} p_{2 i} p_{2 k}$ e $p_{2 j} p_{2 k}$. Foram conservados os níveis zero e seis dos pontos fatoriais e o nível três do ponto central; introduziu-se úm fator multiplicativo a nos níveis dos pontos axiais, tendo-se mantido a distância relativa entre eles. Esse fator multiplicativo a possibilita a anulação dos elementos desejados, em $S$.

Com a introdução do fator multiplicativo $\alpha$, não se con- 
seguiu solução satisfatóría, para a ortogonalização do delineamento, através do modelo com os polinómicos $p_{1 m}$ e $p_{2 m}$.

\subsection{Aplicação de modelo de regressão polinomial quadrätica, com variä-} vel reduzida

$$
\text { Utilizou-se o modelo geral sugerido em BOX e WILSON }
$$

(1951, p. 36), em que a variável genérica $x_{m}$ é agora uma variável reduzida e os componentes quadräticos puros ficam ortogonais à mëdia através de uma reparametrização.

$$
\begin{aligned}
y_{i j k}=b_{0}^{\prime} & +b_{1 i} x_{i}+b_{1 j} x_{j}+b_{1 k} x_{k}+b_{2 i}\left(x_{i}^{2}-\sum x_{i}^{2} / N\right)+b_{2 j}\left(x_{j}^{2}-\Sigma x_{j}^{2} / N\right)+ \\
& +b_{2 k}\left(x_{k}^{2}-\sum x_{k}^{2} / N\right)+b_{1 i 1 j} x_{i} x_{j}+b_{1 i 1 k} x_{i} x_{k}+b_{1 j i k} x_{j} x_{k}+e_{i j k} .
\end{aligned}
$$

onde $b_{0}^{\prime}=b_{0}+\left(\sum x_{m}^{2} / N\right)\left(b_{2 i}+b_{2 j}+b_{2 k}\right)$.

Em termos da variável reduzida $x_{m}$, os pontos fatoriais tẽm coordenadas $\stackrel{+}{-3}$, o ponto central tem coordenada zero e os axiais, con-

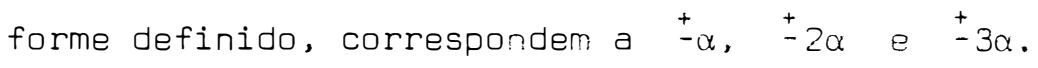

Colocando em forma de matriz, obtém-se:

$$
Y=X R+\varepsilon \quad .
$$

Pelas características do delineamento e do modelo, a matriz $\left[X^{\prime} X\right]$, não-singular, tem os elementos fora da diagonal principal iguais a zero, com exceção dos relativos aos produtos $W$ dos termos quadráticos puros.

$$
\text { Tendo-se que }\left(\Sigma x_{m}^{2} / N\right)=\left(72+28 \alpha^{2}\right) / 27 \text {, os valores de }
$$


$\left(x_{m}^{2}-\Sigma x_{m}^{2} / N\right)$ são dados a seguir:
a) 8 pontos fatoriais
$: \quad\left(171-28 \alpha^{2}\right) / 27$
b) 13 pontos com uma coordenada zero: $\left(-72-28 \alpha^{2}\right) / 27$
c) 2 axiais ${ }^{+} \alpha$
$: \quad\left(-72-\alpha^{2}\right) / 27$
d) 2 axiais $\pm 2 a$
$: \quad\left(-72+80 \alpha^{2}\right) / 27$
e) 2 axiais $\stackrel{+}{-3 a}$
$:\left(-72+215 \alpha^{2}\right) / 27$

Efetuando os produtos correspondentes aos componentes $b_{2 i}$

e $b_{2 j} b_{2 i}$ e $b_{2 k}, b_{2 j}$ e $b_{2 k}$, chega-se à expressão

$$
W=(216 / 729)\left(-98 \alpha^{4}-504 \alpha^{2}+1539\right),
$$

que deve ser tornada igual a zero.

$$
\text { Resolvendo a equação, chega-se ao valor } \alpha=\sqrt{2,1526} \text {. Os }
$$

pontos axiais do delineamento Guadalupe ortogonal estão, portanto, loca lizados nas distáncias aproximadas $\pm 1,467 ; \pm 2,934$ e $\pm 4,402$; os pontos fatoriais mantêm as coordenadas $\stackrel{+}{-3}$. Para efeitos práticos, dependendo do

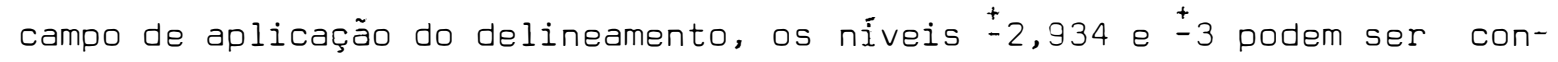
siderados iguais.

o Guadalupe ortogonal, com o mesmo número total de pontos que o original, passou a ter nove níveis em vez de sete; dois deles correspondem à projeção, sobre os eixos, das coordenadas dos pontos fatoriais. Mudando a origem dos valores dos níveis, os pontos fatoriais têm coordenadas em 1,402 e 7,402; 0 ponto central em 4,402; e os pontos axiais em 0 e 8,804; I,468 e 7,336; e 2,935 e 5,869. 


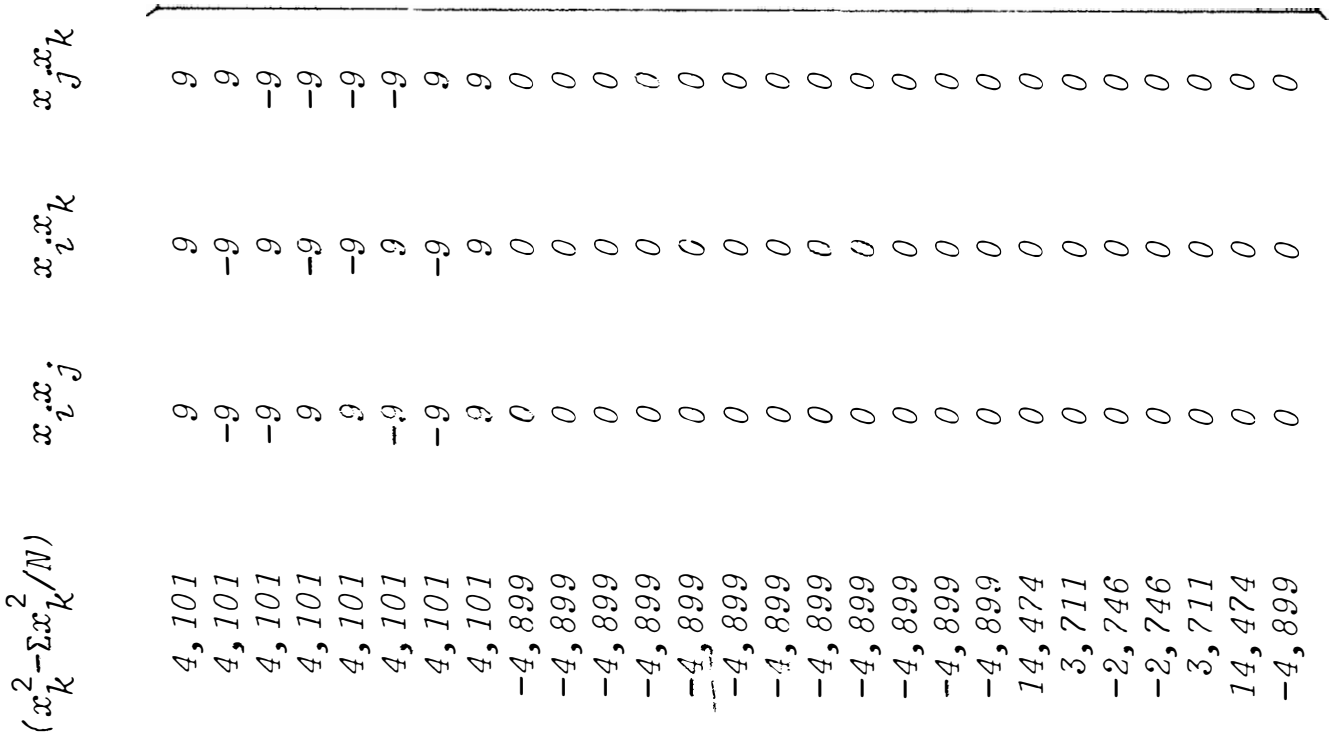

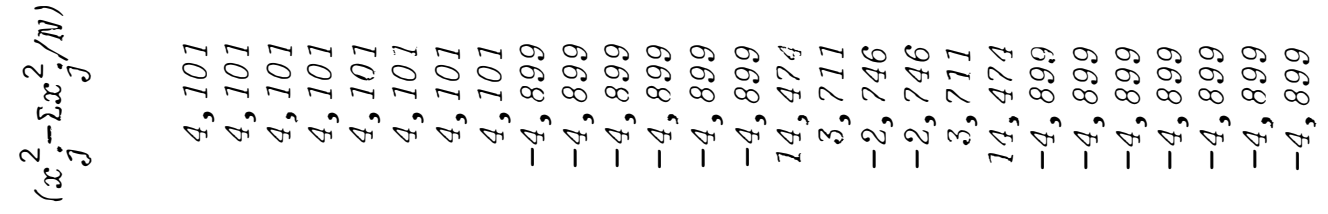

¿.4

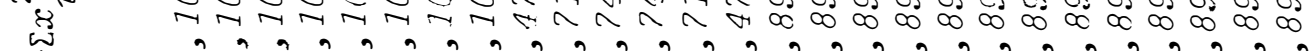

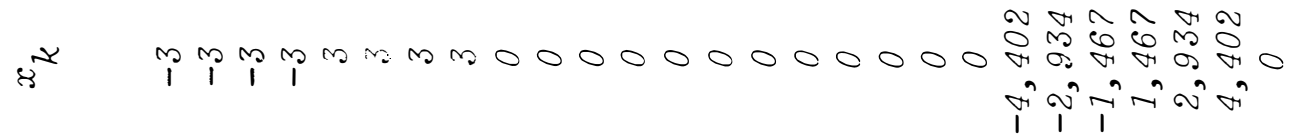

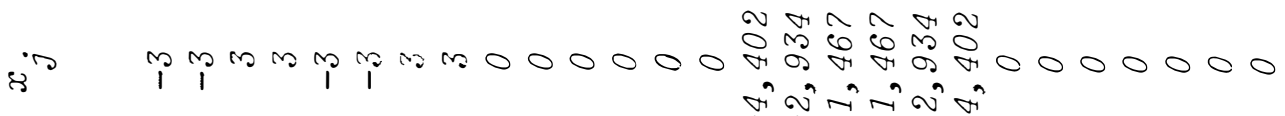
जी

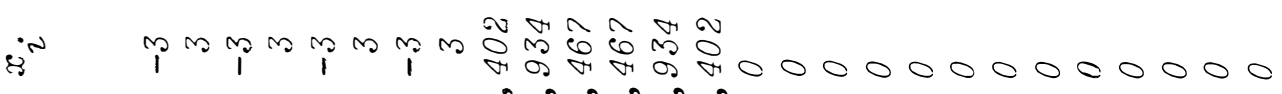
जी १ंगेंते के

$8^{\circ}$ 


$$
X \text { è a matriz das variáveis independentes; suas linhas }
$$

são indicadas por $X_{i j k}^{\prime}$, correspondentes a cada combinação de níveis dos fatores $i, j, k$; suas colunas são designadas por $X_{b}$. Como a segunđa, a terceìra e a quarta coluna correspondem à matriz $D$ do delineamento, neste caso formada com os níveis das variáveis reduzidas, foi omitida a coluna referente às coordenadas de cada ponto.

A partir da matriz $X$ constroem-se $\left(X^{\prime} X\right)$, não-singu lar, e sua inversa $\left(X^{\prime} X\right)^{-1}$.

$\left(X^{\prime} X\right)=\left[\begin{array}{cccccccccccc}27 & 0 & 0 & 0 & 0 & 0 & 0 & 0 & 0 & 0 \\ 0 & 132,267 & 0 & 0 & 0 & 0 & 0 & 0 & 0 & 0 \\ 0 & 0 & 132,267 & 0 & 0 & 0 & 0 & 0 & 0 & 0 \\ 0 & 0 & 0 & 132,267 & 0 & 0 & 0 & 0 & 0 & 0 \\ 0 & 0 & 0 & 0 & 908,166 & 0 & 0 & 0 & 0 & 0 \\ 0 & 0 & 0 & 0 & 0 & 908,166 & 0 & 0 & 0 & 0 \\ 0 & 0 & 0 & 0 & 0 & 0 & 908,166 & 0 & 0 & 0 \\ 0 & 0 & 0 & 0 & 0 & 0 & 0 & 648 & 0 & 0 \\ 0 & 0 & 0 & 0 & 0 & 0 & 0 & 0 & 648 & 0 \\ 0 & 0 & 0 & 0 & 0 & 0 & 0 & 0 & 0 & 648 \\ & & & & & & & & & & & \end{array}\right]$

$$
\left(X^{\prime} X\right)^{-1}=10^{-6}\left[\begin{array}{cccccccccc}
37037 & 0 & 0 & 0 & 0 & 0 & 0 & 0 & 0 & 0 \\
0 & 7560 & 0 & 0 & 0 & 0 & 0 & 0 & 0 & 0 \\
0 & 0 & 7560 & 0 & 0 & 0 & 0 & 0 & 0 & 0 \\
0 & 0 & 0 & 7560 & 0 & 0 & 0 & 0 & 0 & 0 \\
0 & 0 & 0 & 0 & 1101 & 0 & 0 & 0 & 0 & 0 \\
0 & 0 & 0 & 0 & 0 & 1101 & 0 & 0 & 0 & 0 \\
0 & 0 & 0 & 0 & 0 & 0 & 1101 & 0 & 0 & 0 \\
0 & 0 & 0 & 0 & 0 & 0 & 0 & 1543 & 0 & 0 \\
0 & 0 & 0 & 0 & 0 & 0 & 0 & 0 & 1543 & 0 \\
0 & 0 & 0 & 0 & 0 & 0 & 0 & 0 & 0 & 1543 \\
& & & & & & & & &
\end{array}\right]
$$


33.

0 vetor $\hat{B}=\left[X^{\prime} X\right]^{-1} X^{\prime} Y$ é expresso por:

$\hat{B}=\left(\begin{array}{l}b_{0}^{\prime} \\ b_{1 i} \\ b_{1 j} \\ b_{1 k} \\ b_{2 i} \\ b_{2 j} \\ b_{2 k} \\ b_{1 i 1 j} \\ b_{1 i 1 k} \\ b_{1 j 1 k}\end{array}\right)=10^{-6}\left(\begin{array}{l}37037 A \\ 7560 \mathrm{~B} \\ 7560 \mathrm{C} \\ 7560 \mathrm{D} \\ 1101 \mathrm{E} \\ 1101 \mathrm{~F} \\ 1101 \mathrm{G} \\ 1543 \mathrm{I} \\ 1543 \mathrm{~J} \\ 1543 \mathrm{~K}\end{array}\right)$

onde

$$
\begin{aligned}
& A=X_{b}^{\prime}, Y \\
& B=X_{b}^{\prime} \underset{1 i}{Y} \quad E=X_{b}^{\prime} \underset{2 i}{Y} \quad H=X_{b_{1 i 1 j}^{\prime}}^{Y} \\
& c=X_{b}^{\prime} \underset{1 j}{Y} \quad E=X_{b}^{\prime} \underset{2 j}{Y} \quad I=X_{b_{1 i 1 k}^{\prime}}^{Y} \\
& D=X_{b}^{\prime} \underset{1 k}{Y} \quad G=X_{b_{2 k}^{\prime}}^{Y} \quad J=X_{b_{1 j 1 k}^{\prime}}^{Y} \text {. }
\end{aligned}
$$

As variâncias dos elementos de $\hat{\beta}$, dadas pelos elementos correspondentes em $\left(X^{\prime} X\right)^{-1} \sigma^{2}$, são as que se seguem:

$$
\begin{aligned}
& \operatorname{Var} b_{0}^{\prime}=0,037037 \sigma^{2} \\
& \operatorname{Var} b_{1 i}=\operatorname{Var} b_{1 j}=\operatorname{Var} b_{1 k}=0,007560 \sigma^{2} \\
& \operatorname{Var} b_{2 i}=\operatorname{Var} b_{2 j}=\operatorname{Var} b_{2 k}=0,001101 \sigma^{2} \\
& \operatorname{Var} b_{1 i 1 j}=\operatorname{Var} b_{1 i 1 k}=\operatorname{Var} b_{1 j 1 k}=0,001543 \sigma^{2} .
\end{aligned}
$$


34.

Os valores observados têm por variáncia:

a) pontos fatoriais : $0,6716 \sigma^{2}$

b) ponto central : $0,1163 \sigma^{2}$

c) pontos axiais simétricos, a partir dos extremos para o centro do delineamento : $0,4670 \sigma^{2} ; 0,1701 \sigma^{2} ; 0,1144 \sigma^{2}$.

No quadro da análise da variáncia, a SQ Regressão fica subdividida em dez parcelas independentes. 
35.

\title{
6. COMPARAÇÁO DE DELINEAMENTOS
}

\subsection{Eficiência do delineamento Guadalupe em relação aos delineamentos fatorial $3 \times 3 \times 3$ e ao composto central original}

\begin{abstract}
A comparação do delineamento Guadalupe é feita, no presente trabalho, em primeiro lugar em relação ao fatorial $3 \times 3 \times 3$, tendo em vista ser ele um delineamento clássico em pesquisa no campo agronómico, para o qual o Guadalupe foi originalmente proposto. Além disso, o fato de terem ambos o mesmo nümero de pontos experimentais torna de grande interesse prático a comparação.

Pelo critério de PIMENTEL GOMES e CAMPOS (1972), aplicado a ensaios de adubação, o delineamento Guadalupe deve ter seus níveis trazidos para a mesma amplitude dos níveis do fatorial; eles, que vão de -3 a +3 , em variáveis reduzidas, devem ser divididos por 3 para serem comparáveis aos do fatorial, cuja amplitude vai de -1 a +1 . Nesse caso, a variáncia da estimativa dos coeficientes lineares $b_{1 m}$ ficará multiplicada por 9 e a dos quadráticos puros $b_{2 m}$ e dos relativos às interações, representados todos por $\widehat{b}_{1 i 1 j}$, serä multiplicada por 81.
\end{abstract}


Para o delineamento Guadalupe,

$$
\begin{aligned}
& V\left(\hat{b}_{1 m}\right)=0,010000 \sigma^{2} ; V\left(\hat{b}_{2 m}\right)=0,003725 \sigma^{2} ; V\left(\hat{b}_{1 i 1 j}\right)=0,001543 \sigma^{2} \\
& \text { No fatorial } 3 \times 3 \times 3, \\
& V\left(\hat{b}_{1 m}\right)=0,055556 \sigma^{2} ; V\left(\hat{b}_{2 m}\right)=0,166667 \sigma^{2} ; V\left(\hat{b}_{1 i 1 j}\right)=0,083333 \sigma^{2}
\end{aligned}
$$

Aplicando o ajuste, as variàncias do Guadalupe se tornam:

$$
v\left(b_{1 m}\right)=0,090000 \sigma^{2} ; V\left(\hat{b}_{2 m}\right)=0,3017250^{2} ; V\left(b_{1 i 1 j}\right)=0,124983 \sigma^{2} .
$$

Isto mostra que o fatorial $3 \times 3 \times 3$ é mais eficiente que o delineamento Guadalupe, para ensaios com fertilizantes.

Sendo um tipo de delineamento composto central, o Guadalupe foi também comparado ao composto central original, com quinze tratamentos, cujas variāncias são as que se seguem:

$$
V\left(\hat{b}_{1 m}\right)=0,062500 \sigma^{2} ; V\left(\hat{b}_{2 m}\right)=0,090277 \sigma^{2} ; V\left(\hat{b}_{1 i 1 j}\right)=0,125000 \sigma^{2}
$$

Usando o critério de Pimentel Gomes e Campos, após correção para número de tratamentos e para escala, as variàncias dos estimadores dos componentes, no delineamento Guadalupe, em relação ao composto central, se transformam em:

$$
V\left(\hat{b}_{1 m}\right)=0,040500 \sigma^{2} ; V\left(\hat{b}_{2 m}\right)=0,033942 \sigma^{2} ; V\left(\hat{b}_{1 i 1 j}\right)=0,014059 \sigma^{2}
$$

Em ensaios de adubação o Guadalupe apresenta-se, assim, mais eficiente que o composto central original.

Pelo critério de BOX e WILSON (1951), a variància das estimativas dos parâmetros deve ser corrigida para número de pontos do delineamento, quando necessário, e para escala, através do segundo momento em relação à média. Isto vai corresponder a que as variàncias de $b_{1 m}$ se- 
jam multiplicadas pelo valor desse segundo momento, e as variàncias das estimativas dos quadráticos, puros ou não, sejam multiplicadas pelo quadrado desse valor.

No delineamento Guadalupe, corrigido para escala, têm-se as variàncias:

$$
V\left(b_{1 m}\right)=0,0370370^{2} ; V\left(\hat{b}_{2 m}\right)=0,051097 \sigma^{2} ; V\left(\hat{b}_{1 i 1 j}\right)=0,021166 \sigma^{2}
$$

Para o fatorial $3 \times 3 \times 3 \mathrm{com}$ o ajustamento, as variáncias se tornam:

$$
V\left(b_{1 m}\right)=0,037037 \sigma^{2} ; V\left(b_{2 m}\right)=0,074074 \sigma^{2} ; V\left(b_{1 i 1 j}\right)=0,037037 \sigma^{2}
$$

Portanto, na área de ensaios industriais, para os quais, segundo Box e Wilson, o critério havia apresentado resultados razoáveis, - Guadalupe se mostra mais eficiente que o fatorial $3 \times 3 \times 3$.

Corrigindo pela medida proposta por Box e Wilson, as variāncias, no composto central, são dadas por:

$$
V\left(\hat{b}_{1 m}\right)=0,066667 \sigma^{2} ; V\left(\hat{b}_{2 m}\right)=0,1027150^{2} ; V\left(\hat{b}_{1 i 1 j}\right)=0,1422220^{2}
$$

As no Guadalupe, já corrigidas para medida em (1') e agora também para nümero, em relação ao composto central, vēm a seguir:

$$
V\left(\hat{b}_{1 m}\right)=0,066667 \sigma^{2} ; V\left(\hat{b}_{2 m}\right)=0,091962 \sigma^{2} ; V\left(\hat{b}_{1 i 1 j}\right)=0,038097 \sigma^{2}
$$

Comparando-as, vê-se que o delineamento Guadalupe é mais eficiente que o composto central original, para utilização em experimentos no campo industrial ou afins. 
38.

\subsection{Eficiência do delineamento Guadalupe ortogonal em relação ao Gua- dalupe original, ao fatorial $3 \times 3 \times 3$ e ao composto central original}

O delineamento Guadalupe ortogonal tem as seguintes variàncias das estimativas dos parâmetros lineares e quadräticos:

$$
v\left(\hat{b}_{1 m}\right)=0,007560 \sigma^{2} ; V\left(b_{2 m}\right)=0,001101 \sigma^{2} ; V\left(b_{1 i 1 j}\right)=0,001543 \sigma^{2}
$$

A fim de comparä-10, pelo critērio de PIMENTEL GOMES e CAMPOS (1972), aos delineamentos mencionados, as variâncias, corrigidas para medida e, se for o caso, para número de pontos, vêm a seguir.

Para comparação com o Guadalupe original, com variàncias em (1), as coordenadas dos pontos do Guadalupe ortogonal devem ser divididas por 1,4673; portanto, as variàncias são multiplicadas por 2,1530; 4,6353 e 4,6353, respectivamente.

$$
v\left(b_{1 m}\right)=0,016276 \sigma^{2} ; V\left(b_{2 m}\right)=0,005103 \sigma^{2} ; V\left(b_{1 i 1 j}\right)=0,007152 \sigma^{2}
$$

o Guadalupe ortogonal é, assim, menos eficiente que o Guadalupe original, para ensaios de adubação.

As coordenadas do Guadalupe ortogonal devem ser divididas

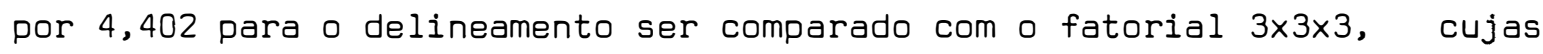
variàncias estão em (2). As variâncias do Guadalupe ortogonal se tornam:

$$
\begin{array}{r}
V\left(\hat{b}_{1 m}\right)=0,146494 \sigma^{2} ; V\left(\hat{b}_{2 m}\right)=0,413415 \sigma^{2} ; V\left(\hat{b}_{1 i 1 j}\right)=0,579383 \sigma^{2} \\
\text { O Guadalupe ortogonal } \dot{e} \text {, portanto, menos eficiente que o }
\end{array}
$$
fatorial $3 \times 3 \times 3$, para o mesmo tipo de ensaios.

Feitas as correções para escala e número de tratamentos, as variāncias no Guadalupe ortogonal, para serem comparadas com as do 
39.

composto central, dadas em (3), são as seguintes:

$$
v\left(\hat{b}_{1 m}\right)=0,065921 \sigma^{2} ; V\left(\hat{b}_{2 m}\right)=0,046508 \sigma^{2} ; V\left(\hat{b}_{1 i 1 j}\right)=0,065179 \sigma^{2}
$$

Então, para ensaios de adubação, pelo critério de Pimentel Gomes e Campos, a precisão da estimativa dos componentes lineares do delineamento Guadalupe ortogonal é cerca de 5\% inferior à dos lineares no composto central; para estimar os componentes quadráticos o Guadalupe ortogonal é, porém, mais eficiente.

As variàncias no Guadalupe ortogonal, com escala ajustada pelo método de Box e Wilson, apresentam os valores:

$$
V\left(\hat{b}_{1 m}\right)=0,037037 \sigma^{2} ; V\left(\hat{b}_{2 m}\right)=0,026424 \sigma^{2} ; V\left(\hat{b}_{1 i 1 j}\right)=0,037037 \sigma^{2}
$$

Comparando-as com as do Guadalupe original (1'), o Guadalupe ortogonal se mostra mais eficiente para estimação dos componentes quadráticos puros e menos para a dos relativos às interações.

Em relação ao fatorial $3 \times 3 \times 3 \quad\left(2^{\prime}\right)$, ele é mais eficiente para estimação dos quadráticos puros e tem mesma eficiència para estimação das interações.

Corrigindo para escala e também para nümero de pontos, para compará-lo ao composto central, (3'), o Guadalupe ortogonal tem as variāncias:

$$
v\left(\hat{b}_{1 m}\right)=0,066667 \sigma^{2} ; V\left(\hat{b}_{2 m}\right)=0,047563 \sigma^{2} ; V\left(\hat{b}_{1 i 1 j}\right)=0,066667 \sigma^{2}, \quad 0
$$

que mostra ser ele mais eficiente que o composto central original, em ensaios no campo industrial. 
40.

\section{EXEMPLO DE APLICAÇÃO}

Para exemplificar a aplicação da metodologia de superfície de resposta polinomial a dados provenientes de experimentos com o delineamento Guadalupe, foi simulado um ensaio de adubação com N, $P$, e K, em milho, tendo-se procurado levar em conta recomendações de PIMENTEL GOMES e ZAGATTO (1967) a respeito de precisão do ensaio e significáncia dos efeitos quadráticos puros.

Os dados foram obtidos a partir da equação fundamental, de segundo grau, que procurou representar uma situação de boa produção no Brasil.

Aos valores esperados de produção, a partir dessa equação, foram adicionados erros provenientes de distribuição normal com coeficiente de variação de 10\%, tirados da Tabela 2 de DIXON e MASSEY (1951, pp. 295 a 304). Foram simulados dados de produção, em quilogramas por hectare, para um experimento em dois blocos completos casualizados. As doses consideradas para cada nutriente foram: 


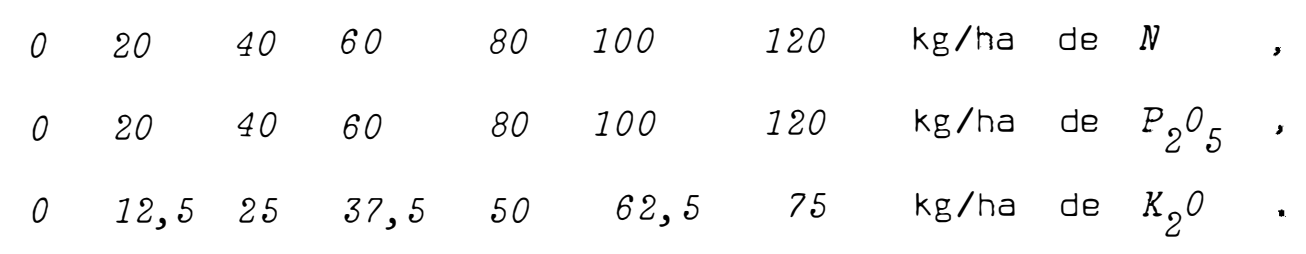

Os dados simulados de produção, relativos à aplicação dos tratamentos especificados, encontram-se nas colunas referentes às repetições I e II.

\begin{tabular}{|c|c|c|c|c|c|c|c|c|c|}
\hline \multicolumn{3}{|c|}{$\begin{array}{l}\text { Níveis de } \\
\text { nutriente }\end{array}$} & \multirow{2}{*}{$\begin{array}{l}\text { Rep I } \\
\mathrm{kg} / \mathrm{ha}\end{array}$} & \multirow{2}{*}{$\begin{array}{l}\text { Rep II } \\
k g / h a\end{array}$} & \multirow{2}{*}{$\begin{array}{l}\text { Média } \\
\mathrm{kg} / \mathrm{ha}\end{array}$} & \multirow{2}{*}{\multicolumn{2}{|c|}{$\begin{array}{l}\text { Valor esperado } \\
\hat{\bar{y}}_{i j k} \\
k g / h a\end{array}$}} & \multirow{2}{*}{\multicolumn{2}{|c|}{$\begin{array}{c}\text { Intervalo de } \\
\text { confiança de } \\
\bar{y}_{i j k} \\
\mathrm{~kg} / \mathrm{ha}\end{array}$}} \\
\hline$N$ & $P$ & $K$ & & & & & & & \\
\hline 0 & 0 & 0 & 764 & 1221 & 992 & 933 & & ( 493, & $1373)$ \\
\hline 6 & 0 & 0 & 2180 & 2491 & 2336 & 2323 & & (1883, & 2723) \\
\hline 0 & 6 & 0 & 2416 & 3120 & 2768 & 2703 & & (2263, & 31431 \\
\hline 6 & 6 & 0 & 4049 & 3394 & 3722 & 3823 & & (3383, & 4263) \\
\hline 0 & 0 & 6 & 1652 & 1697 & 1674 & 1593 & & (1153, & 2033) \\
\hline 6 & 0 & 6 & 2867 & 3304 & 3086 & 3171 & & (27z1, & 3611) \\
\hline 0 & 6 & 6 & 3112 & 3530 & 3321 & 3355 & & (2915, & $3795)$ \\
\hline 6 & 6 & 6 & 4833 & 4333 & 4583 & 4663 & & (4223, & 5103) \\
\hline 0 & 3 & 3 & 2948 & 3463 & 3206 & 3062 & & (2742, & 3382) \\
\hline 1 & 3 & 3 & 3272 & 2906 & 3089 & 3435 & & (3241, & $3629)$ \\
\hline 2 & 3 & 3 & 3893 & 3374 & 3634 & 3748 & & (3602, & $3894)$ \\
\hline 4 & 3 & 3 & 4749 & 4414 & 4582 & 4198 & & $(4052$, & $4344)$ \\
\hline 5 & 3 & 3 & 5286 & 4364 & 4825 & 4334 & & (4140, & $4528)$ \\
\hline 6 & 3 & 3 & 4434 & 4077 & 4256 & 4411 & & (4091, & 4731) \\
\hline 3 & 0 & 3 & 2606 & 3041 & 2824 & 2714 & & (2394, & $3034)$ \\
\hline 3 & 1 & 3 & 3048 & 2985 & 3016 & 3249 & & (3055, & $3443)$ \\
\hline 3 & 2 & 3 & 3806 & 3192 & 3499 & 3678 & & (3532, & 3824) \\
\hline 3 & 4 & 3 & 4389 & 3973 & 4181 & 4222 & & $(4076$ & $4368)$ \\
\hline 3 & 5 & 3 & 4400 & 4512 & 4456 & 4336 & . & $(4142$ & $4530)$ \\
\hline 3 & 6 & 3 & 4293 & 4491 & 4392 & 4345 & & (4025, & $4665)$ \\
\hline 3 & 3 & 0 & 2996 & 3190 & 3093 & 3186 & & (2866, & $3506)$ \\
\hline 3 & 3 & 1 & 3243 & 4195 & 3719 & 3557 & & (3363, & 3751) \\
\hline 3 & 3 & 2 & 3521 & 3907 & 3714 & 3829 & & (3683, & $3975)$ \\
\hline 3 & 3 & 4 & 3947 & 4091 & 4019 & 4079 & & (3933, & $4225)$ \\
\hline 3 & 3 & 5 & 4076 & 4284 & 4180 & 4057 & & (3863) & 4251) \\
\hline 3 & 3 & 6 & 4058 & 3951 & 4004 & 3936 & & (3616, & 4256) \\
\hline 3 & 3 & 3 & 4178 & 3370 & 3774 & 4003 & & (3863, & $4143)$ \\
\hline
\end{tabular}


Utilizando a metodologia que se encontra em 4.4, foram estimados os vetores $P^{\prime} Y$ e $\vec{\beta}$, lembrando que se levou em conta, na matriz $S^{-1}$, o número de repetições para cada tratamento, tendo em vista a existência de dois blocos.

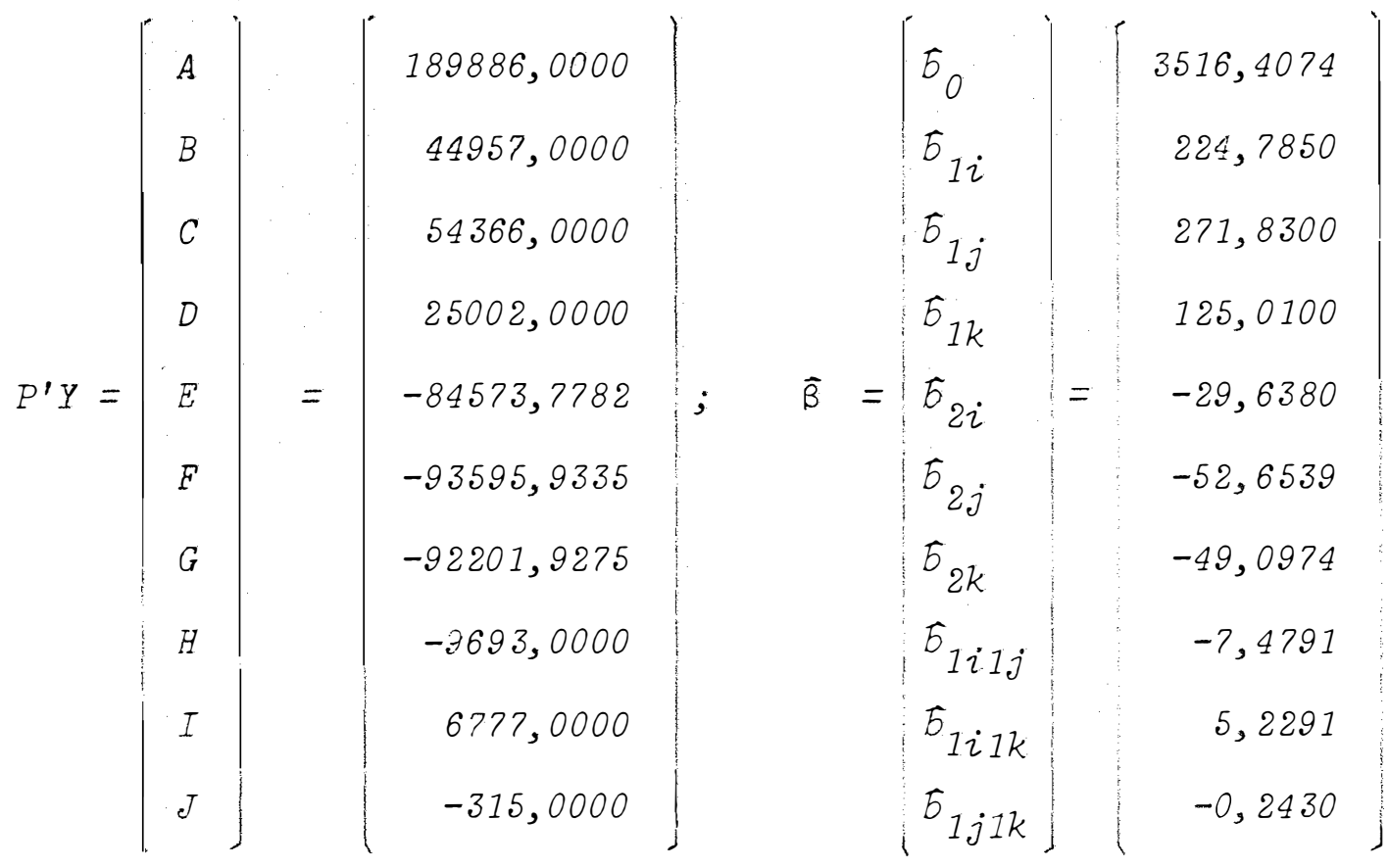

Aplicando a análise da variância aos dados do experimento, chega-se aos resultados que se seguem: 


\begin{tabular}{|c|c|c|c|c|}
\hline F.V. & $S \cdot Q$. & G.L. & Q.M. & $F$ \\
\hline Blocos & 394,74 & 1 & & \\
\hline Média & $667.716 .536,96$ & 1 & & \\
\hline Efeito $\widehat{b}_{1 i}$ & $10.105 .659,24$ & 1 & $10.105 .659,24$ & $84,46^{* *}$ \\
\hline Efeito $b_{1 j}$ & $14.778 .309,78$ & 1 & $14.778 .309,78$ & $123,51^{* *}$ \\
\hline Efeito $\widehat{b}_{1 k}$ & $3.125 .500,02$ & 1 & $3.125 .500,02$ & $26,12^{\star *}$ \\
\hline Ef. $\left(\widehat{b}_{2 i}+\widehat{b}_{2 j}+\widehat{b}_{2 k}\right)$ & $11.961 .663,48$ & 3 & $3.987 .221,16$ & $33,32^{* *}$ \\
\hline Efeito $\widehat{b}_{1 i 1 j}$ & $72.494,92$ & 1 & $72.494,92$ & \\
\hline Efeito $\bar{b}_{1 i 1 k}$ & $35.437,61$ & 1 & $35.437,61$ & \\
\hline Efeito $\hat{b}_{1 j 1 k}$ & 76,54 & 1 & 76,54 & \\
\hline (Regressão) & $(707.795 .678,53)$ & (10) & & \\
\hline Desvios da Regressão & $1.691 .849,47$ & 17 & $99.520,55$ & \\
\hline (Tratamentos) & $(709.487 .528,00)$ & (27) & & \\
\hline Erro & $3.110 .841,26$ & 26 & $119.647,74$ & \\
\hline Total & $712.598 .764,00$ & 54 & & \\
\hline
\end{tabular}

$$
C \cdot V \cdot=9,8 \%
$$

A soma de quadrados relativa aos desvios da regressão não foi significativa, o que mostra a boa adaptação do modelo quadrátiCo.

Calculando a soma de quadrados de cada componente quadrático, ajustada para os dois restantes, foram obtidos os seguintes resultados: 
S.Q. $\hat{b}_{2 i}$ (ajust.) $=471.545,20 ; F_{(1 ; 26)}=3,94($ sign. a $10 \%)$

S.Q. $\tilde{b}_{2 j}$ (ajust.) $=1.488 .372,37 ; F_{(1 ; 26)}=12,43^{* *}$

S.Q. $\widehat{b}_{2 k}$ (ajust.) $=1.294 .111,86 ; F_{(1 ; 26)}=10,81^{* *}$.

Embora não significativo aos níveis habituais de probabilidade, mas levando em conta que, nos ensaios de adubação, o crescimento da resposta ao nitrogènio não é somente linear, resolveu-se manter, na equação, o coeficiente $\hat{b}_{2 i}$ do efeito quadrático. Foram conservados também os outros efeitos de segundo grau, relativos às interações.

Com os elementos de $\widehat{B}$ pode-se escrever a equação polinomial da superfície de resposta, em termos dos polinómios $P_{1 m}$ e $P_{2 m}$.

$$
\begin{aligned}
\hat{y}_{i j k}=3516,4 & +224,8 p_{1 i}+271,8 p_{1 j}+125,0 p_{1 k}-29,64 p_{2 i}- \\
& -52,65 p_{2 j}-49,10 p_{2 k}-7,48 p_{1 i 1 j}+ \\
& +5,23 p_{1 i 1 k}-0,24 p_{1 j 1 k} .
\end{aligned}
$$

Passando para as variäveis $x_{m}$, a fim de mais facilmente realizar a análise econômica do experimento, a equação se transforma em:

$$
\begin{aligned}
\hat{y}_{i j k}=933,2+ & 409,4 x_{i}+610,9 x_{j}+404,6 x_{k}-29,64 x_{i}^{2}-52,65 x_{j}^{2}- \\
& -49,10 x_{k}^{2}-7,48 x_{i} x_{j}+5,23 x_{i} x_{k}-0,24 x_{j} x_{k} \cdot \\
& \text { Com qualquer das equações podem-se çalcular os valores }
\end{aligned}
$$

esperados $\overline{\bar{y}}_{i j k}$, que se encontram nä página 41 , bem como os intervalos de confiança de $\bar{y}_{i j k}$.

A procura do ponto crítico da superfície de resposta, através do estudo da diferencial de segunda ordem, levou ao encontro de ponto de máximo, o que possibilita a análise econômica dos dados experi- 
mentais pelo método usual.

A estimação das quantidades econōmicas de nutrientes foi realizada tomando-se a relação entre os preços de cada nutriente e do produto, com estimação somente por ponto e não por intervalo.

A expressão geral da receita líquida é dada por:

$$
L=\left(\hat{y}_{i j k}-\hat{y}_{000}\right) P_{y}-\left(x_{i} P_{i}+x_{j} P_{j}+x_{k} P_{k}\right)-C F,
$$

onde $P_{y}$ é o preço médio do produto, pago ao produtor, subtraindo-se os custos variäveis de colheita, por unidade de produto; $P_{i}, P_{j}$ e $P_{k}$ são os preços dos nutrientes, ajustados para o acréscimo dos custos de transporte e de colocação do adubo no solo, dos juros correspondentes ao capital empatado e dos subsídios recebidos na compra do adubo; CF são custos fixos do serviço do trator, da adubadeira e da mão de obra.

o valor máximo da receita líquida obtém-se pela solução do sistema de equações:

$$
\frac{\partial L}{\partial x_{i}}=0 ; \quad \frac{\partial L}{\partial x_{j}}=0 ; \quad \frac{\partial L}{\partial x_{k}}=0 \text {, }
$$

que correspondem a

$$
\begin{aligned}
& \frac{\partial y_{i j \underline{k}}}{\partial x_{i}}=\frac{P_{i}}{P_{y}} \quad ; \quad \frac{\partial y_{i j k}}{\partial x_{j}}=\frac{P_{j}}{P_{y}} ; \quad \frac{\partial y_{i j k}}{\partial x_{k}}=\frac{P_{k}}{P_{y}} \\
& \text { Considerando os valores } 120,105 \text { e } 35 \text { para as relações }
\end{aligned}
$$

acima, as coordenadas do ponto de receita líquida máxima são as seguintes:

$$
x_{i}=4,67 ; \quad x_{j}=4,46 ; \quad x_{k}=4,00,
$$

equivalentes, aproximadamente, a 95,90 e $50 \mathrm{~kg} / \mathrm{ha}$ de $N, P_{2} \mathrm{O}_{5}$ e $K_{2} \mathrm{O}$, respectivamente. 


\section{CONCLUSOES}

\subsection{Delineamento Guadalupe original para três fatores}

A aplicação da metodologia de superfície de resposta, através da utilização de um modelo de regressão, com polinômios ortogo nais de $1^{\circ}$ e de $2^{\circ}$ grau em $x$, a experimento planejado com o uso do delineamento Guadalupe, para três fatores, levou aos seguintes resultados e conclusões:

8.1.1. As estimativas de todos os coeficientes de $1^{\circ}$ e de $2^{\circ}$ grau, encontradas pelo mëtodo dos quadrados mínimos, são independentes da estimativa do coeficiente $b_{0}$, correspondente à média.

8.1.2. Somente as estimativas dos coeficientes quadräticos puros estão correlacionadas, e as estimativas das covariāncias entre eles representam cerca de um terço da estimativa de suas variàncias.

8.1.3. As variâncias das respostas estimadas $\hat{y}_{i j k}$ em cada ponto do delineamento, para os níveis utilizados, na escala adotada, são as seguintes: 
a) pontos fatoriais : $0,7638 \sigma^{2}$

b) ponto central : $0,0770 \sigma^{2}$

c) pontos axiais simétricos, a partir dos extremos para o centro do delineamento $\quad: 0,4040 \sigma^{2} ; 0,1478 \sigma^{2} ; 0,0836 \sigma^{2}$.

8.1.4. Para ensaios de adubação, o delineamento Guadalupe é menos eficiente que o fatorial $3 \times 3 \times 3$ e mais eficiente que o composto central original (critério de Pimentel Gomes e Campos).

8.1.5. Para ensaios no campo industrial o delineamento Guadalupe é mais eficiente que o fatorial $3 \times 3 \times 3$ e o composto central original (critério de Box e Wilson).

\subsection{Delineamento Guadalupe ortogonal para três fatores}

8.2.1. A ortogonalização do delineamento Guadalupe, para trés fatores e igual nümero de pontos que o Guadalupe original, através de um modelo de regressão polinomial quadrática aumentou de sete para nove o nümero de níveis de cada fator. Os dois níveis adicionais correspondem à projeção dos pontos fatoriais sobre os eixos.

8.2.2. Os pontos fatoriais e o central permaneceram nas coordenadas $\pm_{-3}$ e zero, respectivamente, e os pontos axiais encontram-se nas distâncias aproximadas $\pm 1,467 ; \pm 2,934$ e $\pm 4,402$.

8.2.3. As variâncias das respostas estimadas $\hat{y}_{i j k}$ em cada ponto do delineamento, para os níveis mencionados, são as que se seguem:
a) pontos fatoriais : $0,6716 \sigma^{2}$
b) ponto central : $0,1163 \sigma^{2}$ 
c) pontos axiais simétricos, a partir dos extremos para o centro do delineamento : $0,4670 \sigma^{2} ; 0,1701 \sigma^{2} ; 0,1144 \sigma^{2}$.

8.2.4. Em ensaios de adubação, o delineamento Guadalupe ortogo nal, com vinte e sete pontos, para três fatores, é menos eficiente que o

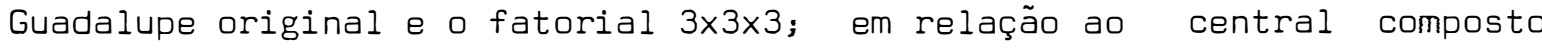
original, o Guadalupe ortogonal é mais eficiente na estimação dos componentes quadráticos.

8.2.5. Em ensaios na ärea industrial, o Guadalupe ortogonal ë mais eficiente que o original ao estimar os componentes quadráticos puros e menos eficiente ao estimar as interações; em relação ao fatorial $3 \times 3 \times 3$, é mais eficiente para estimação dos quadráticos puros e tem mesma eficiência em relação às interações; é ainda mais eficiente que o composto central original. 


\section{SUMMARY}

The development of experimental and treatment designs, that provide a more efficient approach to investigate response surfaces, was initiated by R.A. Fisher, in the agronomic field, and extended to other research areas.

The central composite and the rotatable designs, applicable mainly in the chemical industry, were also extended to other investigation fields.

In the present thesis, the response surface methodology was applied to a type of central composite design, named Guadalupe, for three factors at seven equally spaced levels, with twenty seven treatments. It has a central point, eighteen axial and eight factorial points, with their projections on the axes interval extremes. "

A quadratic polynomial regression was used in the analysis: with orthogonal polynomials of first and second degroe. The estimates of the model parameters, their variances and covariances, the anazy\$s of variance and the tests of hypotheses, the confidence Intervals 
50.

for parameters and expected values are given. Their adaptation to the Guadalupe design is developed.

Comparing with the $3 \times 3 \times 3$ factorial and the central composite designs, the Guadalupe is less efficient than the first and more efficient than the second, when applied to fertilizer experiments, according to PIMENTEL GOMES and CAMPOS (1972) criterion. But it is more efficient in the chemical industry and similas fields of investigation, by BOX and WILSON (1951) criterion,

The Guadalupe design has become orthogonal by imposing conditions when fitting a new quadratic polynomial regression model. The six axial and the center points are still equally spaced. The projections of the factorial points, on the axes, introduce two new levels of each factor, with nine levels on the whole.

By the same criteria, the orthogonalized Guadalupe is less efficient than the original Guadalupe and the $3 \times 3 \times 3$ factorial, and more efficient than the central composite design, in fertilizer trials, but it is the most efficient, in estimating the pure quadratic components, in chemical research.

A simulated $N, P, K$ fertilizer experiment, with conn (maize), is given as an example of using the Guadalupe design in agricultural research, utilizing the response surface methodology developed in the present paper. 
51.

\section{LITERATURA CITADA}

ALVAREZ, R. A. SEgALLA, A.C. PIMENTEL WUTKE \& E.S. FREIRE, 1963. Adubação da cana-de-açủcar VIII. Adubação mineral em solos massapé-salmourão (1957-58). Bragantia. Campinas. 22: 657-675.

ANDERSON, R.L. E T.A.BANCROFT, 1952. Statistical Theomy in Researeh. Nova York, McGraw-Hill. 399 p.

ARRUDA, H.V. de, 1959. Contribuicão para o Estudo da Adubasäo Mineral do Mitho nas Terras Roxas do Municipio de Ribeirão Preto. Piracicaba. ESALQ/USP. 39 p. (Tese de Doutoramento).

BATISTA, L.B., 1978. Delineamento em circulos. Pesq. Agropec. Bras, Brasilia, $13(4): 9-15$.

BOX, G.E.P. E J.S. HUNTER, 1957. Multi-factor experimental designs for exploring response surfaces, Ann, Math. Statist., An Arbor. 28: 195-241.

BOX, G.E.P. e K.B.WILSON, 1951. On the experimental attainment of optimum conditions. JZ. R. Statire. Soc., Londres, B 13: 1-45. 
CONAGIN, A., J. de P.N. JORGE e W.R. VENTURINI, 1969. Delineamentos ex perimentais utilizáveis na experimentação de campo. In: REYNAERT, E.E., Ed. Ia Investigación de Fertilidad de Suelos para la Pro ducción Agricola en la Zona Templada. Montevidéu, IICA-ZC, p. 183$-201$.

CHRISTIANS, N.E., O.P. MARTIN e J.F. WILKINSON, 1979. Nitrogen, phosphorus, and potassium effects on quality and growth of Kentucky bluegrass and creeping bentgrass. Agron. J., Madison, 71: 564-567.

COCHRAN, W.G. e G.M. COX, 1957. Experimental Designs. 2a. ed. Nova York, John Wiley. $611 \mathrm{p}$.

COX, O.R., 1958. Planning of Experiments. Nova York, John Wiley. 308 p.

DAVIES, 0.L., Ed., 1954. The Design and Analysis of Industrial Experiments. Londres, Oliver and Boyd. $636 \mathrm{p}$.

DAVILA, L.E.R., 1979. Algunos diseños para estudios de la superfície de respuesta a la aplicación de fertilizantes. In: Reunião Internacional de Biometria, Região Brasileira. Piracicaba, 1975. Fundação Cargill, p. 131-156.

OTXON, W.J. \& F.J. MASSEY, 1951. Introduction to Statistical Analysis. Nova York, McGraw-Hill. $370 \mathrm{p}$.

FINNEY, D.J., 1945. The fractional replication of factorial arrangements. Ann. Eugen., Londres, 12: 291-301.

FISHER, R.A., 1926. The arrangement of fleld experiments. Jour. Min. Agric., Londres, 33: 503-513. In: SHEWHART, W.A., Ed., 1950. B.A. Fisher: Contributions to Mathematical Statistics. Nova - Yark, John Wiley, p. 17.503-17.513. 
FUZATTO, M.G., W.R. VENTURINI e P.A. CAVALERI, 1970. Estudo técnico-econômico da adubação do algodoeiro no Estado de São Paulo. Campinas, Instituto Agronômico. $15 \mathrm{p}$. (Projeto BNOE/ANOA/CIA. $\mathrm{n}^{8}$ 1).

GILL, J.L., 1968. Design and Analysis of Experiments in the Animal and Medical Sciences, Volume 2. Ames, The Iowa State University Press. $301 \mathrm{p}$.

GRANDAGE, A., 1958. Orthogonal coefficients for unequal intervals. Biometrics. Raleigh, 14: 287-289.

GRAYBILL, F.A., 1961. An Introduction to Linear Statistical Models. Volume 1. Nova York, McGraw-Hill. 463 p.

HEAOY, E.O. e J.L. DILLON, 1961. Agricultural Production Functions, 3a. ed. Ames, The Iowa State Univ. Press. $667 \mathrm{p}$.

HICKS, C.R., 1973. Fundomental Concepts in the Design of Experiments, 2a. ed. Nova York. Holt, Rinehart and Winston. $349 \mathrm{p}$.

HOFFMANN, R. e S. VIEIRA, 1977. Anälise de Regressão. Uma Introdução à Econometria. São Paulo, HUCITEC-EOUSP. $339 \mathrm{p}$.

IGUE, T.. 1968. Interacōes em Grupos de Experimentos de Adubacão do Feijoeiro com N, $P$ e $K$, Segundo o Esquema Fatorial $3 \times 3 \times 3$. Piracicaba, ESALQ/USP. 81 p. (Dissertação de Mestrado).

MALAVOLTA, E., F. PIMENTEL GOMES e outros, 1963. A diagnose foliar na cana de açúcar IV. Piracicaba, ESALQ/USP. 47 p.

MARTINEZ GARZA. A. 1978. Hacia el Incremento de la Froductividad. Mètodos Estadistico - Matemäticos. Chapingo. CEC, Colëgio de Postgraduados. $125 \mathrm{p}$. 
MEAD, R. e D.J. PIKE, 1975. A review of response surface methodology from a biometric viewpoint. Biometrics. Raleigh, 31: 803-851.

MIRANDA, L.T. de, 1966. Resultados de experimentos de adubação e suges. tões para a interpretação baseada na anälise química do solo. In: Cultura e Adubacão do Milho. São Paulo, Instituto Brasileiro de Potassa. $541 \mathrm{p}$.

MITSCHERLICH, E.A., 1909. Das Gesetz des Minimuns und das Gesetz das Abnehmenden Bodenertrages. Landuirtschastliche Jahrbllcher. Berlin, 38: $537-552$.

MYERS, R.H., 1971. Response Surface Methodology. Boston, Allyn and Bacon. $246 \mathrm{p}$.

NOGUEIRA, I.R., 1979. Método geral para obtenção de tabelas de polinômios ortogonais. Rev. Agricultura. Piracicaba, 54 (4): 269-279.

PAEZ, G. e T. SILVA, 1975. Delineamento dos Experimentos de Adubacão. Brasilia, EMBRAPA, O.P.0. 55 p.

PIMENTEL GOMES, F. e A.G. ZAGATTO, 1967. Aspectos econömı́cos da adubação. In: MALAVOLTA, E. Manual de Quimica AgricoZa-Adubos e Adubasão. São Paulo, Ed. Agr. Ceres. 606 p.

PIMENTEL GOMES, F., 1969. Novos aspectos do estudo económico de adubação. Fertilité. Paris, 34: 3-9.

PIMENTEL GOMES, F. e H. CAMPOS, 1972. The efficiency of factorial $3^{3}$ designs as compared to a central composite rotatable design. Potash Review, Berna. Fevereiro. 3 p. 
ROJAS, B.A., 1963. The San Cristobal designs for fertilizer experiments. In: WILlIAM, J.R., Ed. Proc. Int. Soc. of Sugar Cane Technologists. Eleventh Congress, Mauritius. Nova York, Elsevier Publ. Comp., p. 197$-203$.

ROJAS, B.A., 1972. The orthogonalized San Cristóbal design. In: HENDER-SON, M.T., Ed. Proc. Int. Soc. of Sugar Cane Technologists. Fourteenth Congress, Nova Orleans. Louisiana, Franklin Press Inc., p. $1085-1093$.

SEARLE, S.R., 1971. Linear Models. Nova York, John Wiley. 532 p.

SNEDECOR, G.W. e W.G. COCHRAN, 1967. Statistical Methods. 6a. ed. Ames, The Iowa State University Press. 593 p.

SPILLMAN, W.J. e E. LANG, 1924. The Law of Diminishing Retwons. Chicago, World Book Co. 178 p.

STAVROU, J. e F.B. CADY, 1967. Counfounding the triple cube response surface design to reduce block size. Soil Sci. Soc. Amer. Proc., Madison, 31: 126-128.

STRAUSS, E., 1951. Experimentos de adubação da zona canavieira de Pernambuco. Anais da Terceira Reunião Brasileira de Ciências do Solo, p. $336-446$.

TRAMEL, T.E., 1957. A suggested procedure for agronomic-economic fertilizer experiments. In: BAUM, E.L., E.O. HEADY, J.T. PESEK e C.G. HILDRETH, Ed. Economic and Technical Analysis of Fertilizer Innovations and Resource Use. Ames, The Iowa State College Press, p. 168-175 . 
56.

VANDERLIP, R.L. e J. PESEK, 1970. Nitrate accumulation in smooth bromegrass (Bromus inermis, Leyss.): I. Effects of applied $N, P$, and $K$. Agron. J., Madison, 62:491-494.

VOSS, R. e J. PESEK, 1967. Yield of corn grain as affected by fertilizer rates and environmental factors. Agron. J., Madison, 59:567-572.

WISHART, J., 1938. Growth rate determination in nutrition studies with the bacon pig, and their analysis. Biometrika. Londres, 30:16-28.

YATES, F., 1935. Complex experiments. Jl. R. Statist. Soc., Londres, B2:181-247. In: YATES, F., 1970. Experimental Design. Londres, Griffin, p. 71-117. 